\title{
ALMA Resolves the Nuclear Disks of Arp 220
}

\section{Citation}

Scoville, Nick, Lena Murchikova, Fabian Walter, Catherine Vlahakis, Jin Koda, Paul Vanden Bout, Joshua Barnes, et al. 2017. "ALMA Resolves the Nuclear Disks of Arp 220." The Astrophysical Journal 836 (1): 66. https://doi.org/10.3847/1538-4357/836/1/66.

\section{Permanent link}

http://nrs.harvard.edu/urn-3:HUL.InstRepos:41381714

\section{Terms of Use}

This article was downloaded from Harvard University's DASH repository, and is made available under the terms and conditions applicable to Other Posted Material, as set forth at http:// nrs.harvard.edu/urn-3:HUL.InstRepos:dash.current.terms-of-use\#LAA

\section{Share Your Story}

The Harvard community has made this article openly available.

Please share how this access benefits you. Submit a story.

\section{Accessibility}




\title{
ALMA Resolves the Nuclear Disks of Arp 220
}

\author{
Nick Scoville $^{1}$, Lena Murchikova ${ }^{1}$, Fabian Walter ${ }^{2}$, Catherine Vlahakis ${ }^{3}$, Jin Koda $^{4}$, Paul Vanden Bout ${ }^{3}$, \\ Joshua Barnes ${ }^{5,6}$, Lars Hernquist ${ }^{7}$, Kartik Sheth $^{8}$, Min Yun ${ }^{5}$, David Sanders ${ }^{6}$, Lee Armus ${ }^{9}$, Pierre Cox ${ }^{10,11}$, \\ Todd Thompson $^{12,13}$, Brant Robertson ${ }^{14}$, Laura Zschaechner ${ }^{2}$, Linda Tacconi ${ }^{15}$, Paul Torrey ${ }^{16,17}$,

\begin{abstract}
We present 90 mas $(37 \mathrm{pc})$ resolution ALMA imaging of Arp 220 in the CO (1-0) line and continuum at $\lambda=2.6 \mathrm{~mm}$. The internal gas distribution and kinematics of both galactic nuclei are well resolved for the first time. In the west nucleus, the major gas and dust emission extends out to 0 ". 2 radius ( $74 \mathrm{pc})$; the central resolution element shows a strong peak in the dust emission but a factor of 3 dip in the $\mathrm{CO}$ line emission. In this nucleus, the dust is apparently optically thick $\left(\tau_{2.6 \mathrm{~mm}} \sim 1\right)$ at $\lambda=2.6 \mathrm{~mm}$ with a dust brightness temperature of $\sim 147 \mathrm{~K}$. The column of interstellar matter at this nucleus is $N_{\mathrm{H} 2} \geqslant 2 \times 10^{26} \mathrm{~cm}^{-2}$, corresponding to $\sim 900 \mathrm{gr} \mathrm{cm}^{-2}$. The east nucleus is more elongated with radial extent 0 !" 3 or $\sim 111 \mathrm{pc}$. The derived kinematics of the nuclear disks provide a good fit to the line profiles, yielding the emissivity distributions, the rotation curves, and velocity dispersions. In the west nucleus, there is evidence of a central Keplerian component requiring a central mass of $8 \times 10^{8} M_{\odot}$. The intrinsic widths of the emission lines are $\Delta v(\mathrm{FWHM})=250$ (west) and 120 (east) $\mathrm{km} \mathrm{s}^{-1}$. Given the very short dissipation timescales for turbulence $\left(\lesssim 10^{5}\right.$ years), we suggest that the line widths may be due to semicoherent motions within the nuclear disks. The symmetry of the nuclear disk structures is impressive, implying the merger timescale is significantly longer than the rotation period of the disks.
\end{abstract}

Key words: galaxies: active - galaxies: individual (Arp 220) - galaxies: starburst - Galaxy: evolution ISM: clouds - ISM: molecules

\section{Introduction}

Galactic merging is a key process in the early growth and evolution of massive galaxies and in determining their structural morphology. In the era of precision cosmology, these processes remain a major uncertainty in understanding the present makeup of the visible universe. Many aspects of the evolution of merging, gas-rich nuclear disks are poorly constrained and only approximately understood in terms of the physical processes and the hierarchy of their importance. The radial mass and star-formation distributions, the physical conditions (density, temperature, and cloud structures), and the evolution associated with feedback from starbursts (SBs) and active galactic nuclei (AGNs) remain poorly understood. And yet, all of these are vital to predicting the ultimate fate or product of the mergers (e.g., the resulting galactic morphologies) and the mode(s) of star formation and AGN fueling.
Ultraluminous infrared galaxies (ULIRGs) are the most extreme SB galaxies. The first complete sampling of the local universe yielded 22 ULIRGs at $z<0.1$ from the IRAS all-sky survey with $L_{1-1000 \mu \mathrm{m}}>10^{12} L_{\odot}$ (Sanders et al. 1988). At high redshift when the rate of collisions of the galaxies was higher (Rodriguez-Gomez et al. 2015) and galaxies were more gasrich, they were much more abundant (Caputi et al. 2007; Le Floc'h et al. 2009; Magnelli et al. 2013).

Resolved studies of the merging processes must rely on the sample of local galaxies. The follow-up ground-based optical imaging reveals virtually all of the local ULIRGs to be merging galaxies or postmerging systems (Armus et al. 1987; Sanders et al. 1988; Sanders \& Mirabel 1996). During the merging of gasrich galaxies, the original interstellar matter (ISM; presumably distributed in extended galactic-scale disks) sinks rapidly to the center of the merging system due to dissipation of kinetic energy in the shocked gas and torques generated by the offset stellar and 
Table 1

Fluxes

\begin{tabular}{|c|c|c|c|c|c|c|}
\hline Source & Frequency & $\alpha_{2000^{\mathrm{a}}}$ & $\delta_{2000^{\mathrm{a}}}$ & Total Flux & Peak Flux & Peak $T_{B}$ \\
\hline Arp 220 West & 112.26 & $15: 34: 57.224$ & $23: 30: 11.48$ & 32.1 & $13.1 \pm 0.05$ & 147.2 \\
\hline Lines & & & & $\left(\mathrm{Jy} \mathrm{km} \mathrm{s}^{-1}\right)$ & $\left(J y \mathrm{~km} \mathrm{~s}^{-1}\right.$ beam $\left.^{-1}\right)$ & (K) \\
\hline Arp 220 West & $\mathrm{CO}(1-0)$ & $15: 34: 57.224$ & $23: 30: 11.49$ & 47.3 & $3.33 \pm 0.01$ & $187^{\mathrm{b}}$ \\
\hline
\end{tabular}

Notes. Peak position and fluxes obtained from 2D Gaussian fits using the IDL routine CURVEFIT. Gaussian component sizes are listed in Table 2. Uncertainties in the continuum and line fluxes obtained from the uncertainties in the Gaussian component fitting do not include calibration uncertainties.

${ }^{a}$ Peak derived from two-dimensional Gaussian fit (Table 2).

${ }^{\mathrm{b}}$ Peak brightness temperature from the spectral cube within $\pm 600 \mathrm{~km} \mathrm{~s}^{-1}$ of the systemic velocity for the line or from the continuum images of the dust.

gaseous bars (e.g., Barnes \& Hernquist 1992, 1996). The starformation rates in the ULIRGs are typically 10-100 times higher per unit mass of ISM compared to quiescent disk galaxies. The ULIRG-starburst activity is likely driven by the concentration of gas in nuclear regions and dynamical compression of this gas in supersonic shocks.

Among the ULIRGs, Arp 220 is probably the most frequently cited example, having luminosity $L_{\mathrm{IR}}=1.91 \times 10^{12}$ $L_{\odot}$ (Sanders et al. 2003; Armus et al. 2009). Here we adopt a luminosity distance of $D_{\mathrm{L}}=87.9 \mathrm{Mpc}$ and angular size distance $D_{\mathrm{A}}=85.0 \mathrm{Mpc}$ (Armus et al. 2009). Near-infrared imaging shows two galactic nuclei separated by 1 ". 0 or $412 \mathrm{pc}$ (Scoville et al. 1998). CO imaging at 0.5 resolution uncovers two counterrotating disks with radii $\sim 100 \mathrm{pc}$ and dynamical masses $\sim 2 \times 10^{9} M_{\odot}$ for each disk (Scoville et al. 1997, 1998; Sakamoto et al. 1999; Downes \& Eckart 2007). Much of the nuclear ISM is very dense $\left(>10^{4-5} \mathrm{~cm}^{-3}\right)$ and at high temperature ( $>75$ K; Sakamoto et al. 1999; Downes \& Eckart 2007; Greve et al. 2009; Matsushita et al. 2009; Rangwala et al. 2011; Wilson et al. 2014; Scoville et al. 2015b).

Both nuclei are optically thick at $\lambda<600 \mu \mathrm{m}$. Wilson et al. (2014) derive dust optical depths $\tau_{434 \mu \mathrm{m}}=5.3$ and 1.7 for the west and east nuclei, respectively, implying that for the west nucleus $\tau_{1 \mathrm{~mm}} \sim 1$. These high optical depths imply that the nuclear disk structures are best probed at $\lambda>600 \mu \mathrm{m}$. Shorter wavelength observations may not penetrate the outer dust photospheres of the nuclei unless the structures are tilted to the line of sight. The fact than any near-infrared radiation can be seen from the nuclei is a clear indication that the dust is in a disk-like distribution tilted to the line of sight.

Here, we present 90 mas $(37 \mathrm{pc}$ ) resolution ALMA imaging of the inner region of Arp 220 in the CO (1-0) line and the $2.6 \mathrm{~mm}$ continuum (108-114 GHz, ALMA Band 3), providing excellent resolution and sensitivity for imaging the molecular gas and the long-wavelength dust continuum. The east and west nuclei are internally resolved for the first time. The dust continuum provides an independent and linear probe of the overall ISM mass (Scoville et al. 2015a, 2015b).

\section{ALMA Observations and Data Reduction}

These ALMA Cycle 3 long baseline observations in the CO (1-0) line were obtained in 2015 November for project \#2015.1.00113.S. (We are also scheduled to obtain CO (2-1) and (3-2) high- and low-resolution imaging, but those data will probably not be available until the end of 2017.) In view of the major increase in resolution provided by the high-resolution CO (1-0) data, publication of these data is important, and we proceed here with those preliminary results.

The observations discussed here were in receiver Band 3; the correlator was configured in the time division mode (TDM) with four spectral windows. Each window had a full bandwidth of $1875 \mathrm{MHz}$ with $1.95 \mathrm{MHz}$ resolution spectral channels. One window was configured to observe the redshifted CO (1-0) line at a rest frequency of $115.2712 \mathrm{GHz}$; the remaining three spectral windows were centered at $113.253 \mathrm{GHz}, 103.073 \mathrm{GHz}$, and $101.139 \mathrm{GHz}$ to image the dust continuum emission and ${ }^{13} \mathrm{CO}$ and $\mathrm{C}^{18} \mathrm{O}$. The latter will be presented in our later publication with the other bands.

The observations were done in a very extended configuration with baselines up to $11 \mathrm{~km}$, providing the maximum resolution presently available with ALMA. For this telescope configuration, good flux recovery is expected out to scales of $\sim 0$ ". 4 , but extended emission with spatial size greater than this will be at least partially resolved out. These observations thus probe only the inner nuclei of Arp 220. The data were taken with $4012 \mathrm{~m}$ antennas, and the total integration time was 30 minutes (excluding calibrations).

Following delivery of data products, the data were rereduced and imaged using the Common Astronomy Software Applications package (CASA). Self-calibration was done to improve the dynamic range. The images were made with the parameters Robust $=0,1$, and 2; only the Robust $=1$ images are used here. We cleaned the images with no continuum subtraction, noted the line and absorption free channels, and then created a continuum image using those channels. The continuum subtraction was then done in the image plane.

Channel averaging over eight of the original channels was done to reduce noise, resulting in data with a velocity resolution of $40 \mathrm{~km} \mathrm{~s}^{-1}$ without serious compromise relative to the intrinsic line width $\left(\Delta v_{\mathrm{FWHM}}=100-200 \mathrm{~km} \mathrm{~s}^{-1}\right.$; see Section 5). The $1 \sigma$ (rms) sensitivities are as follows: an rms noise of $0.6 \mathrm{mJy}$ beam $^{-1}$ in $40 \mathrm{~km} \mathrm{~s}^{-1}$ channels in the lower sideband at $\sim 110 \mathrm{GHz}$, and $0.8 \mathrm{mJy} \mathrm{beam}^{-1}$ in $40 \mathrm{~km} \mathrm{~s}^{-1}$ channels in the upper sideband at $114 \mathrm{GHz}$.

The velocities given here are $v_{\text {radio }}=c z /(1+z)=$ $c\left(\nu_{\text {rest }}-\nu\right) / \nu_{\text {rest }}$ relative to the $\mathrm{LSR}$ (not $v_{\text {opt }}=c z=$ $\left.c\left(\nu_{\text {rest }}-\nu\right) / \nu\right)$. The Arp 220 observations were centered on 
Table 2

Gaussian Source Fits

\begin{tabular}{|c|c|c|c|c|c|c|c|c|}
\hline \multirow[b]{2}{*}{ Source } & \multicolumn{4}{|c|}{ Gaussian Fit } & \multicolumn{4}{|c|}{ Deconvolved } \\
\hline & Peak Flux & $\begin{array}{l}\text { Major } \\
\left({ }^{\prime \prime}\right)\end{array}$ & $\begin{array}{l}\text { Minor } \\
\left({ }^{\prime \prime}\right)\end{array}$ & $\begin{array}{l}\text { PA } \\
\left({ }^{\circ}\right)\end{array}$ & $\begin{array}{c}\text { Major } \\
\left({ }^{\prime \prime}\right)\end{array}$ & $\begin{array}{l}\text { Minor } \\
\left({ }^{\prime \prime}\right)\end{array}$ & $\begin{array}{l}\text { PA } \\
\left({ }^{\circ}\right)\end{array}$ & $\begin{array}{c}T_{B} \\
(\mathrm{~K})\end{array}$ \\
\hline Arp 220 West & $11.87 \pm 0.02$ & $0.13 \pm 0.01$ & $0.12 \pm 0.01$ & 50.7 & 0.12 & 0.11 & 64.1 & 167.0 \\
\hline Arp 220 East & $2.46 \pm 0.02$ & $0.24 \pm 0.02$ & $0.15 \pm 0.01$ & 49.2 & 0.24 & 0.14 & 50.7 & 33.2 \\
\hline$C O(1-0)$ Lines & $\left(\mathrm{Jy} \mathrm{km} \mathrm{s}^{-1}\right)$ & & & & & & & \\
\hline Arp 220 West & $2.96 \pm 0.01$ & $0.37 \pm 0.01$ & $0.33 \pm 0.02$ & 164.5 & 0.36 & 0.32 & 162.7 & $\cdots$ \\
\hline Arp 220 East & $1.44 \pm 0.01$ & $0.54 \pm 0.02$ & $0.25 \pm 0.01$ & 46.2 & 0.54 & 0.24 & 46.5 & $\cdots$ \\
\hline
\end{tabular}

Note. Sizes (FWHM) and major-axis PA estimates obtained from 2D Gaussian fits using the IDL routine CURVEFIT. The deconvolved sizes were obtained using the IDL routine MAX_LIKELIHOOD. The uncertainties in the parameters were the formal errors from the Gaussian fitting. For all of the observations, the synthesized beam was $0.08 \times 0 . \prime 10$ at $\mathrm{PA}=25^{\circ} .7$.

$z=0.018486$, implying $c z=v_{\mathrm{opt}}=5542 \mathrm{~km} \mathrm{~s}^{-1}$ and $v_{\text {radio }}=$ $5441 \mathrm{~km} \mathrm{~s}^{-1}$ (Sanders et al. 1991). The derived systemic velocities of the nuclei are $v_{\text {radio }}=5337$ (west) and $5431 \mathrm{~km} \mathrm{~s}^{-1}$ (east) (Table 4).

Table 1 lists the measured source fluxes and peak brightness temperatures for both the continuum and the $\mathrm{CO}(1-0)$ line, and Table 2 contains the results of two-dimensional Gaussian fits to each of the sources. The total recovered $\mathrm{CO}$ line flux from the two nuclei is $45.4+27.4=72.8 \mathrm{Jy} \mathrm{km} \mathrm{s}^{-1}$. This is $19 \%$ of the total single-dish CO (1-0) line flux measured by Sanders et al. (1991) and Solomon et al. (1997).

\section{Continuum Emission}

\subsection{Infrared Dust Emission}

A brief background for understanding the far-infrared dust emission is warranted as a preamble to our analysis below. For this discussion we might visualize the massive dust concentrations in the nuclei of Arp 220 as spherical, with density decreasing outward. The dust has an opacity that increases steeply at shorter wavelengths (i.e., $\kappa_{\nu} \propto \nu^{1.8}$ in the farinfrared/submillimeter regime). The enormous dust column densities in the Arp 220 nuclear sources imply that the dust will be optically thick well into the far-infrared.

As one views these sources from the outside, the depth from which the observed, emergent radiation is emitted will depend strongly on the wavelength of observation, since at each wavelength one sees into the photosphere at $\tau_{\lambda} \simeq 1$. At longer wavelengths the dust opacity is less, so the emergent radiation will come from deeper within the optically thick cloud.

The dust at each radius is also likely to be in radiative equilibrium with the luminosity (which originates from massive stars in the nuclear starburst or from a central AGN). As long as these luminosity sources are centrally concentrated, the dust temperatures must increase at smaller radii.

Thus it should be anticipated - as long as the dust is optically thick into the far-infrared, the longer wavelength observations will tend to probe high-temperature dust closer to the central energy sources-that the long wavelengths see deeper into the enveloping dust. It would appear counterintuitive that longer wavelength observations probe hotter regions in the nuclei at smaller radii, but because of the opacity falloff at longer wavelengths, this is in fact the case.
There is a cautionary corollary: lower angular resolution observations will tend to include a larger fraction of the cooler dust at large radii, and these outer regions will be less optically thick (if the gas and dust density decreases outward). In fact, one must recognize that there is no single wavelength at which one can say the source is optically thin or thick. The dust column (and optical depth) increases as the line of sight passes closer to the center; therefore the "apparent" (or average) optical depth one infers from an observation is dependent on the angular resolution or beam size of the observation.

\subsection{Continuum Spectra}

In Table 3, we list the radio and infrared continuum measurements done at $<0$ ". 6 resolution, sufficient to separate the two nuclei (Figure 1). The total integrated fluxes and peak flux per beam are given, along with the peak brightness temperature calculated from the peak flux per beam at each of the different frequencies. It should be noted that the synthesized beam sizes vary between 0 ". 6 and 0 ". 08 . In most cases, the interferometric integrated fluxes will recover emission on scales up to $\sim 3 \times$ the beam size, but emission on larger scales will not be fully recovered. The brightness temperatures determined from the peak flux at each frequency also refer to variable source radii ranging from 0 ." 05 to 0 ." 3 , corresponding to 20 to $123 \mathrm{pc}$.

In Figure 2 the fluxes are plotted, and the spectral indexes for each frequency interval are shown. At low frequencies $(\nu<40 \mathrm{GHz})$, the spectral index $\left(\alpha\right.$ in $\left.S_{\nu} \propto \nu^{\alpha}\right)$ is negative, and the emission is predominantly nonthermal synchrotron. At $\nu \geqslant 200 \mathrm{GHz}$ the dust emission dominates, and the spectral indexes are ranging from $\alpha=2$ (optically thick and a single dust temperature) to steeper $(\alpha>2$ to $\lesssim 4)$ if there are substantial contributions from optically thin dust. In this frequency range, the maximum spectral index will be $\sim 3.8$, corresponding to optically thin emission with a typical Galactic ISM dust opacity coefficient of $\beta=1.8 \pm 0.1$ (Planck Collaboration 2011a). In Figure 2 on the right, the spectral indexes at the highest frequency are 2.4 (west) and 3.2 (east), indicating that most of the emission is from optically thick dust with a small addition of optically thin emission (most obvious in the east nucleus where the optical depths are not so high). 
Table 3

Continuum Fluxes

\begin{tabular}{|c|c|c|c|c|c|}
\hline $\begin{array}{l}\nu_{\text {obs }} \\
(\mathrm{GHz})\end{array}$ & $\begin{array}{l}\text { Beam } \\
\left(" \times{ }^{\prime \prime}\right)\end{array}$ & $\begin{array}{l}\text { Total Flux } \\
\text { (mJy) }\end{array}$ & $\begin{array}{l}\text { Peak Flux } \\
\text { (mJy/beam) }\end{array}$ & $\begin{array}{c}\text { Peak } T_{B}{ }^{\mathrm{a}} \\
\text { (K) }\end{array}$ & Reference \\
\hline \multicolumn{6}{|c|}{ West Nucleus } \\
\hline 4.70 & $0.60 \times 0.43$ & 114.6 & $89.5 \pm 0.7$ & $1.92 \times 10^{4}$ & Barcos-Muñoz et al. (2015) \\
\hline 5.95 & $0.38 \times 0.28$ & 94.3 & $73.3 \pm 1.0$ & $2.38 \times 10^{4}$ & Barcos-Muñoz et al. (2015) \\
\hline 7.20 & $0.38 \times 0.28$ & 89.5 & $60.4 \pm 0.7$ & $1.34 \times 10^{4}$ & Barcos-Muñoz et al. (2015) \\
\hline 32.5 & $0.08 \times 0.06$ & 33.4 & $6.5 \pm 0.8$ & 1570 & Barcos-Muñoz et al. (2015) \\
\hline 112.3 & $0.10 \times 0.08$ & 29.0 & $13.1 \pm 0.5$ & $121^{\mathrm{b}}$ & Scoville et al. 2016 \\
\hline 229.4 & $0.30 \times 0.30$ & 106 & $79.0 \pm 2.0$ & 90 & Downes \& Eckart (2007) \\
\hline 341.8 & $0.55 \times 0.40$ & 328 & $261 \pm 2$ & 12.4 & Scoville et al. (2015b) \\
\hline 347.9 & $0.46 \times 0.39$ & 384 & $283 \pm 0.4$ & 80 & Martin et al. (2016) \\
\hline 349.6 & $0.25 \times 0.21$ & 360 & $360 \pm 50$ & 160 & Sakamoto et al. (2008) \\
\hline 691 & $0.36 \times 0.20$ & 1810 & $1150 \pm 11$ & 40.9 & Wilson et al. (2014) \\
\hline \multicolumn{6}{|c|}{ East Nucleus } \\
\hline 4.70 & $0.60 \times 0.43$ & 92.4 & $61.8 \pm 0.5$ & $1.33 \times 10^{4}$ & Barcos-Muñoz et al. (2015) \\
\hline 5.95 & $0.38 \times 0.28$ & 81.4 & $49.0 \pm 0.7$ & $1.59 \times 10^{4}$ & Barcos-Muñoz et al. (2015) \\
\hline 7.20 & $0.38 \times 0.28$ & 73.2 & $36.0 \pm 0.4$ & 7980 & Barcos-Muñoz et al. (2015) \\
\hline 32.5 & $0.08 \times 0.06$ & 30.1 & $4.1 \pm 0.5$ & 988 & Barcos-Muñoz et al. (2015) \\
\hline 112.3 & $0.10 \times 0.08$ & 16.1 & $3.1 \pm 0.5$ & $15^{\mathrm{b}}$ & Scoville et al. 2016 \\
\hline 341.8 & $0.55 \times 0.40$ & 157 & $111 \pm 3$ & 5.3 & Scoville et al. (2015b) \\
\hline 347.9 & $0.46 \times 0.39$ & 192 & $115 \pm 0.4$ & 15 & Martin et al. (2016) \\
\hline 349.6 & $0.25 \times 0.21$ & 190 & $190 \pm 50$ & 52 & Sakamoto et al. (2008) \\
\hline 691 & $0.36 \times 0.20$ & 1510 & $800 \pm 11$ & 28.4 & Wilson et al. (2014) \\
\hline
\end{tabular}

Notes. Includes only continuum data at $<0$ " 6 resolution. Scoville et al. (2016) is this paper.

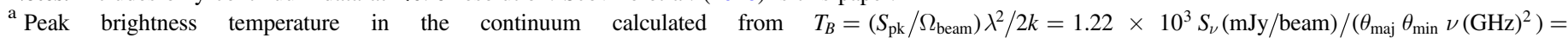
$1.36 S_{\nu}(\mathrm{mJy} /$ beam $\left.) \lambda(\mathrm{cm})^{2}\right) /\left(\theta_{\text {maj }} \theta_{\text {min }}\right)$ where the FWHM beam sizes $\theta$ are in arcsec.

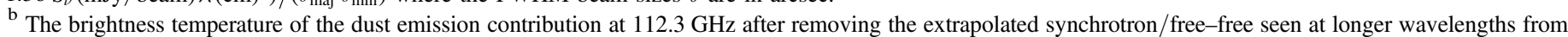
the observed $T_{B}$ of $163 \mathrm{~K}$ (west) and $39 \mathrm{~K}$ (east).

\subsection{Dust Emission Fluxes and Brightness Temperatures}

At $112.3 \mathrm{GHz}$, the continuum emission is a mixture of synchrotron and dust emission. If we extrapolate the $32 \mathrm{GHz}$ flux measurements (Barcos-Muñoz et al. 2015) with a spectral index $\alpha=-0.60$ determined between 7 and $32 \mathrm{GHz}$ (see Figure 2), then the expected nonthermal and free-free contribution at $112.3 \mathrm{GHz}$ will be $\sim 0.47$ of the $32.5 \mathrm{GHz}$ fluxes, implying 15.8 and $3.1 \mathrm{mJy}^{\text {beam }}{ }^{-1}$ in the west nucleus and 14 and $1.9 \mathrm{mJy}$ beam $^{-1}$ in the east nucleus. Subtracting these contributions from the observed fluxes, we obtain estimates for the dust continuum fluxes of 13 and $10 \mathrm{mJy}$ beam $^{-1}$ (west) and 1.8 and $1.2 \mathrm{mJy} \mathrm{beam}^{-1}$ (east), at 112.3 GHz. These dust-only fluxes are used to calculate the revised dust emission brightness temperatures given in parentheses in Table 3 for $112.3 \mathrm{GHz}-121 \mathrm{~K}$ in the west nucleus and $15 \mathrm{~K}$ in the east on the scale 90 mas diameter or $19 \mathrm{pc}$ radius. The peak brightness temperatures in the CO (1-0) emission are 187 and $175 \mathrm{~K}$, respectively (see Table 1); these peak line brightness temperatures are seen at larger radii (see Figure 3).

\subsection{West Nucleus Luminosity}

The $147 \mathrm{~K}$ brightness temperature of the $112.3 \mathrm{GHz}$ dust emission seen on the west nucleus is unexpectedly high, given the previous submillimeter observations $(691 \mathrm{GHz})$ of Wilson et al. (2014) that indicated $T_{B}=181 \pm 27 \mathrm{~K}$ on the west nucleus and an optical depth of $\tau_{434 \mu \mathrm{m}} \simeq 5$.3. If the dust opacity varies with the standard Galactic power law $\nu^{1.8}$, then the optical depth should be $\sim 0.038 \times 5.3 \sim 0.2$ at $112.6 \mathrm{GHz}$. The estimated $147 \mathrm{~K}$ brightness temperature clearly requires a higher optical depth of $\tau_{2.6 \mathrm{~mm}} \simeq 1$.

It is interesting to note that the implied dust photosphere, optically thick out to $\lambda \sim 2.6 \mathrm{~mm}$, with dust temperature $T_{\mathrm{D}} \simeq 200 \mathrm{~K}$ and radius $\simeq 15 \mathrm{pc}$, must radiate

$$
\begin{aligned}
L_{\text {west } 200 \mathrm{~K} \text { dust }}= & 4 \pi R^{2} \sigma T^{4} \\
= & 6.35 \times 10^{11} L_{\odot} \\
& \times(R / 15 \mathrm{pc})^{2}(T / 200 \mathrm{~K})^{4} .
\end{aligned}
$$

An identical estimate, $L_{\mathrm{West}}=6.3 \times 10^{11} L_{\odot}$, was obtained by Wilson et al. (2014) from ALMA dust continuum measurements at $\lambda=445 \mu \mathrm{m}$. This corresponds to $33 \%$ of the total IR luminosity $1.91 \times 10^{12} L_{\odot}$ of Arp 220 (Sanders et al. 2003; Armus et al. 2009)—all originating from $R \leqslant 15 \mathrm{pc}$.

Based on their $860 \mu \mathrm{m}$ continuum measurements at 0 ". 23 resolution, Sakamoto et al. (2008) obtain lower limits on the luminosity $\sim 3 \times 10^{11} L_{\odot}$ for the west nucleus. They argue that the derived luminosity is very sensitive to the adopted source size, varying as $R^{-6}$. Our estimate given above is based on a resolved source size and a dust temperature of $\sim 200 \mathrm{~K}$, based on a determination that the dust is nearly optically thick. It therefore is not just a lower limit but rather an estimate of the actual luminosity, albeit uncertain to a factor of 2 .

For a geometrically thin, optically thick disk, the emitting surface area is $\sim 2 \pi R^{2}$, so for the same $T_{\mathrm{D}}$ the emergent luminosity would be a factor of 2 lower than for Equation (1). 

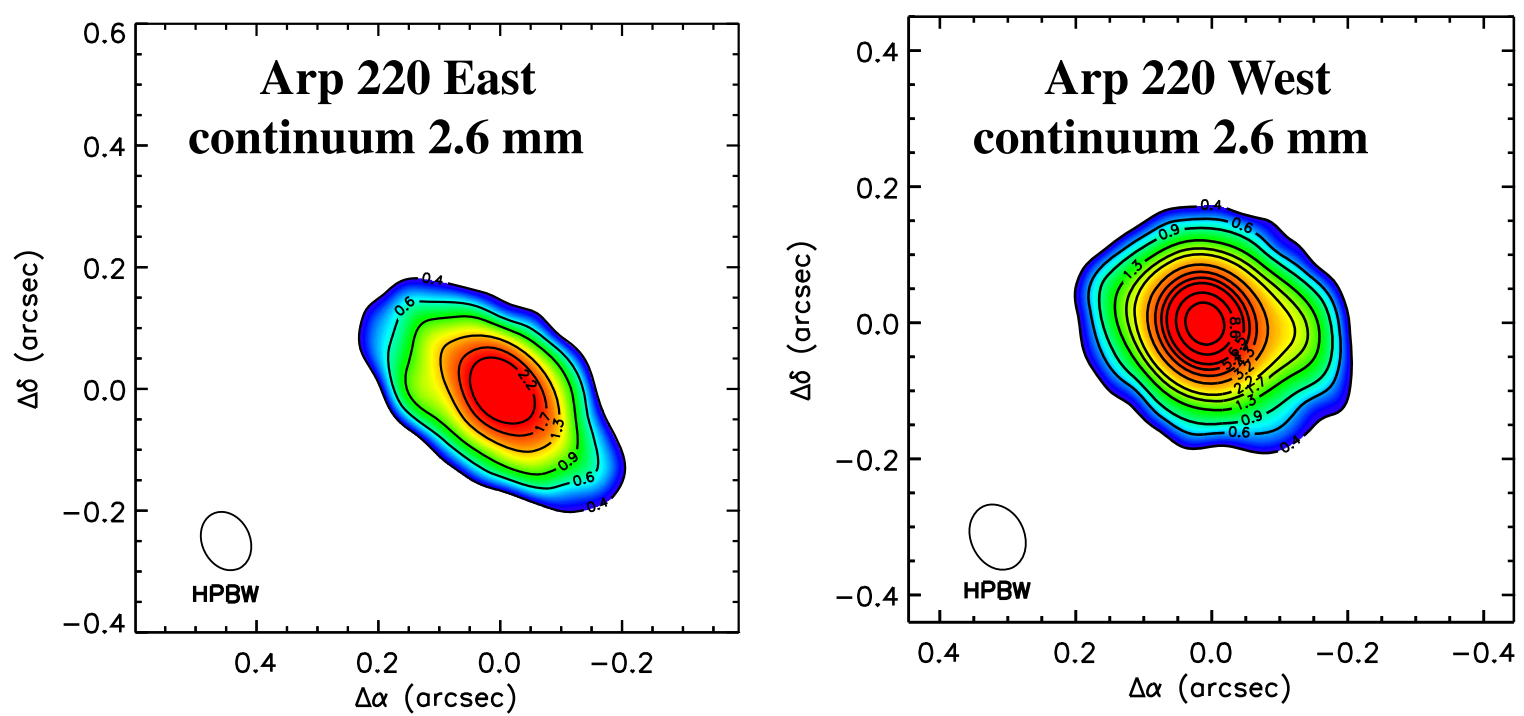

Figure 1. The $2.6 \mathrm{~mm}$ continuum distribution on the east and west nuclei is shown at $0.08 \times 00^{\prime \prime} 1$ resolution $(33 \times 41 \mathrm{pc})$. The peak values are 3.1 (east) and $13.1 \mathrm{mJy}$ beam $^{-1}$ (west). Coordinate offsets are relative to the $2.6 \mathrm{~mm}$ continuum peaks (Table 1), and the contours are mJy beam ${ }^{-1}$.

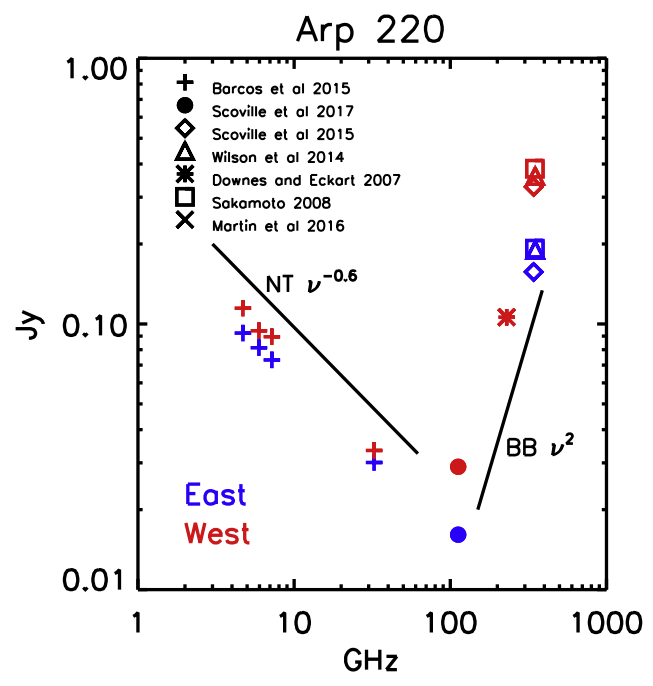

Figure 2. Continuum fluxes measured at high resolution for the east and west nuclei of Arp 220 (Table 3). The expected power laws for nonthermal synchrotron emission (NT) and Rayleigh-Jeans blackbody (BB) emission are shown for reference. At long wavelengths, the dominant emission is nonthermal synchrotron emission with flux in the range $\nu^{-0.5 \text { to }-0.7}$; at $\nu>100 \mathrm{GHz}$ the dominant emission is the dust continuum with spectra varying between $\nu^{2}$ in the optically thick limit and $\nu^{\sim 3.8}$ in the optically thin limit. The dust emission at $\nu>100 \mathrm{GHz}$ is optically thick in the smallest aperture measurements and a combination of both optically thick and thin emission in larger aperture measurements.

It is interesting to note that essentially all estimates of the infrared luminosity in ULIRGs assume a spherical, that is, isotropic source. If the infrared is in fact emitted from a thin disk that is optically thick, these luminosity estimates should be increased by a factor of $1 / \cos i$.

A considerably lower temperature $T=45 \mathrm{~K}$ is derived from fitting the total far-infrared SED of Arp 220 (Sanders et al. 1991), implying that, at the shorter wavelengths near the $70 \mu \mathrm{m}$ IR peak, one is sampling colder dust in a more extended photosphere. This sampling of colder dust at shorter wavelengths is as anticipated in Section 3.1 for an optically thick dust cloud: the optical depth is higher at shorter wavelengths. Hence, the $\tau \simeq 1$ surface (from which the observed photons at shorter wavelengths originate) will be at larger radius and lower $T$ (assuming a centrally heated source).

A major virtue of the longer wavelength observations reported here is the ability to penetrate the optically thick dust envelope and probe the inner regions of heavily obscured luminosity sources. The peak of the $200 \mathrm{~K}$ blackbody emission $\left(L_{\nu}\right)$ is at $\lambda=26 \mu \mathrm{m}$, but the original sources of luminosity (young stars or a central AGN) undoubtedly emit their energy at much shorter wavelengths in the visible, UV, and X-rays.

Since the observations place an upper limit of $\sim 200 \mathrm{~K}$ on the dust temperature at $R=15 \mathrm{pc}$, this implies that the luminosity originating from smaller radii cannot be much larger than $6 \times 10^{11} L_{\odot}$-otherwise the dust would be hotter. If there is an AGN in the western nucleus, its power must be less than this. The remainder of the $\sim 1.9 \times 10^{12} L_{\odot}$ total luminosity, or about $1.3 \times 10^{12} L_{\odot}$, must originate from more distributed star formation at $R>15 \mathrm{pc}$ (36 mas radius) and the east nucleus.

\subsection{West Nucleus ISM Column Densities and Mass}

We use the observed dust opacity $\left(\tau_{2.6 \mathrm{~mm}} \simeq 1\right)$ to estimate the column density of the west nucleus point source assuming that the dust there has properties similar to the general ISM dust observed in the Galaxy. Scoville et al. (2015a) empirically calibrated the long-wavelength dust emission from Herschel SPIRE with the CO (1-0) ISM masses for 28 local star-forming galaxies and 12 ULIRG galaxies and with Planck measurements of the submillimeter dust opacity in the Milky Way. The data are consistent with a single proportionality constant relating the rest-frame $850 \mu \mathrm{m}$ specific luminosity of the dust to the molecular ISM mass. This empirical calibration is

$$
\begin{aligned}
\alpha_{\nu} & \equiv\left\langle L_{\nu_{850 \mu \mathrm{m}}} / M_{\mathrm{mol}}\right\rangle \\
& =(6.7 \pm 1.7) \times 10^{19} \mathrm{erg} \mathrm{s}^{-1} \mathrm{~Hz}^{-1} M_{\odot}^{-1},
\end{aligned}
$$

where $L_{\nu_{850 \mu \mathrm{m}}}$ is the specific luminosity of the dust at $\lambda=$ $850 \mu \mathrm{m}$. The mass $M_{\text {mol }}$ includes a contribution for $\mathrm{He}$ and 
Arp 220 East CO (1-0)
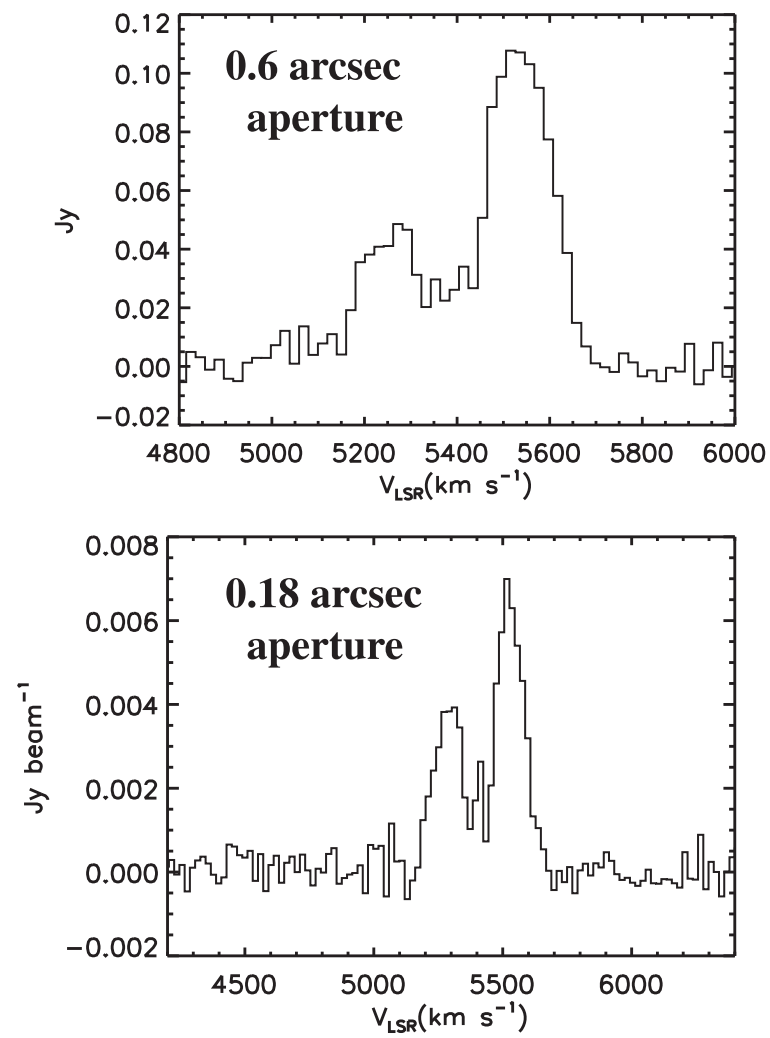

Arp 220 West CO (1-0)
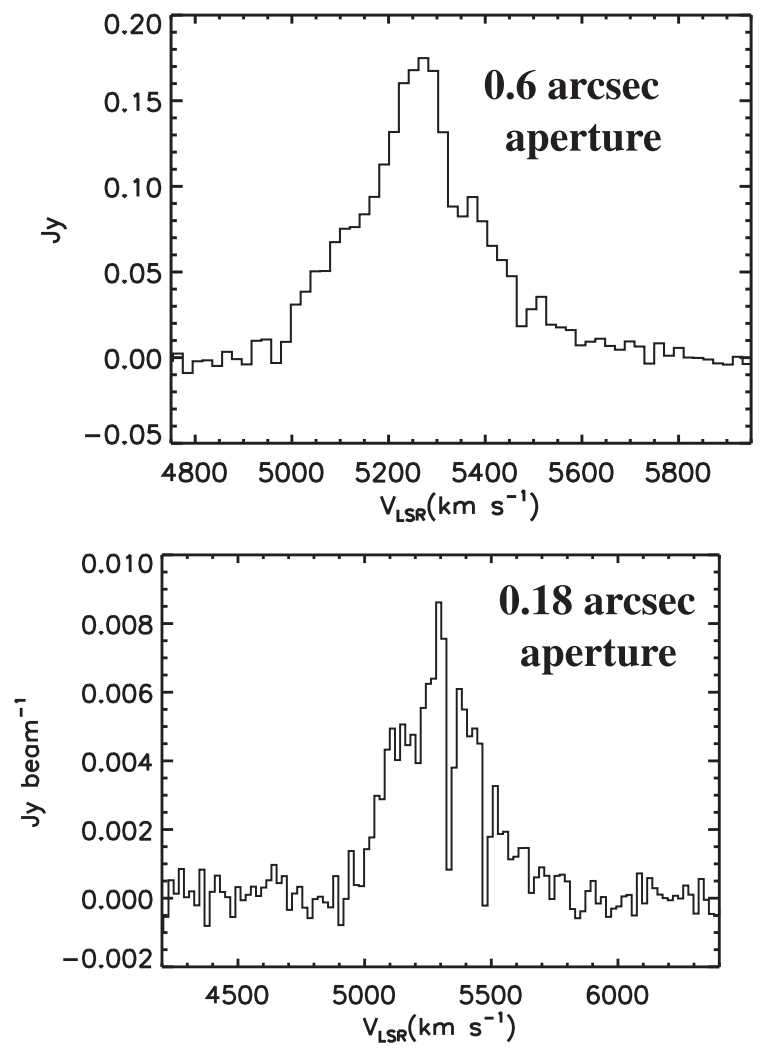

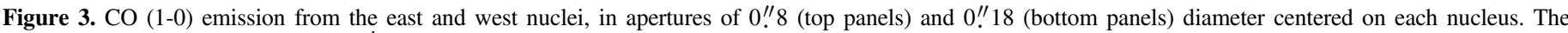

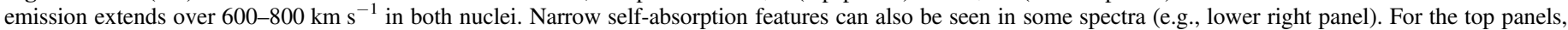

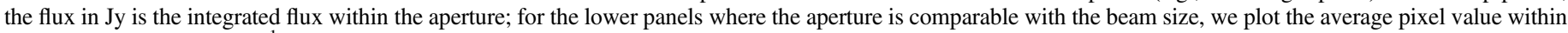
the aperture (i.e., Jy beam ${ }^{-1}$ ). Note that in the bottom spectra the velocity range is much larger.

heavier atoms, and the mass derivation employed a single standard Galactic $\mathrm{CO}-\mathrm{H}_{2}$ conversion factor.

For an optically thin mass sheet of area $A$ and surface density $\Sigma_{\text {mol }}$ ISM, Equation (2) can be recast as

$$
\begin{aligned}
L_{\nu_{850 \mu \mathrm{m}}} / M_{\mathrm{mol}} & =\frac{2 \pi A B_{\nu}\left(T_{\mathrm{D}}\right) \tau_{\nu}}{A \Sigma_{\mathrm{mol}}} \\
& =\frac{2 \pi B_{\nu}\left(T_{\mathrm{D}}\right) \kappa_{\nu} \Sigma_{\mathrm{mol}}}{\Sigma_{\mathrm{mol}}} \\
& =2 \pi B_{\nu}\left(T_{\mathrm{D}}\right) \kappa_{\nu}, \quad \text { yielding } \\
\kappa_{\nu} & =\frac{\alpha_{\nu}}{2 \pi B_{\nu}\left(T_{\mathrm{D}}\right)} .
\end{aligned}
$$

We use the above calibration of dust optical depth to estimate the column of gas in the nuclear source. The bulk of the ISM dust in the nearby galaxies used for the calibration is at $\sim 25 \mathrm{~K}$ (see Scoville et al. 2015a). Using this temperature in the above equations then implies a dust absorption coefficient at $850 \mu \mathrm{m} \quad \kappa_{850 \mu \mathrm{m}}=8.06 \times 10^{-3} \mathrm{~cm}^{2} \mathrm{gr}^{-1}$, where the mass includes the He contribution. Scaling this opacity coefficient as $\nu^{1.8}$ (Planck Collaboration 2011a), we obtain $\kappa_{2.6 \mathrm{~mm}}=$ $1.03 \times 10^{-3} \mathrm{~cm}^{2} \mathrm{gr}^{-1}$. Putting this in terms of the $\mathrm{H}_{2}$ column density, $\kappa_{2.6 \mathrm{~mm}}=4.51 \times 10^{-27} \mathrm{~N}_{\mathrm{H}_{2}}$. Thus an $\mathrm{H}_{2}$ column of $2.21 \times 10^{26} \mathrm{H}_{2} \mathrm{~cm}^{-2}$ is required in order for $\tau_{2.6 \mathrm{~mm}} \simeq 1 .{ }^{19}$

\footnotetext{
19 Alternatively, the Planck ratio of $\tau_{250 \mu \mathrm{m}} / N_{\mathrm{H}}=2.32 \times 10^{-25} \mathrm{~cm}^{2}$ derived for Milky Way $\mathrm{H}_{2}$ (Planck Collaboration 2011b) translates to $1.45 \times 10^{26} \mathrm{~cm}^{2}$ $\mathrm{H}_{2} \mathrm{~cm}^{-2}$ when adopting the same $\nu^{1.8}$ dependence of the opacity coefficient.
}

Our estimate of the column density is considerably higher than earlier estimates of $N_{\mathrm{H} 2} \sim 10^{25}$ and $3-6 \times 10^{25} \mathrm{~cm}^{-2}$ (Sakamoto et al. 2008; González-Alfonso et al. 2012). Their estimates were lower since they were derived from higher frequency observations and hence should be viewed as lower limits if the dust is optically thick at those wavelengths. Our estimate is also well above the lower limit of $>10^{25} \mathrm{~cm}^{-2}$ derived by Teng et al. (2015) from nondetections in the NuSTAR bands above $20 \mathrm{keV}$.

As an aside, it is interesting to note that the mass column density of ISM $\simeq 900 \mathrm{~g} \mathrm{~cm}^{-2}$ in the western nucleus corresponds to a concrete wall $3-4 \mathrm{~m}$ thick or gold $1 \mathrm{ft}$ thick. This is perhaps the highest ISM column density ever probed by astronomical observations. It corresponds to $A_{V}=2 \times 10^{5}$ mag and would be very Compton thick.

Lastly, we note that $\tau_{2.6} \mathrm{~mm}$ must be $\simeq 1$ across a circular region with $R>10$ pc since the similarity of the observed dust brightness temperature and dust physical temperature requires an areal filling factor of order unity in the central resolution element. The total molecular mass is then $M_{\text {mol }}>1.4 \times 10^{9}$ $M_{\odot}$. Clearly, these estimates are uncertain, given the assumption that the dust has standard interstellar dust properties and abundance, relative to gas in the extreme conditions at the center of Arp 220. The mass so derived is approximately a factor of 2 higher than the dynamical mass in the same region (also uncertain) obtained from the $\mathrm{CO}$ line kinematics (see Section 5).

From the derived $\mathrm{H}_{2}$ column density $N_{\mathrm{H}_{2}}=2.21 \times 10^{26}$ $\mathrm{H}_{2} \mathrm{~cm}^{-2}$ and line-of-sight path length of $30 \mathrm{pc}$, we infer a mean 
Arp 220 East CO (1-0)
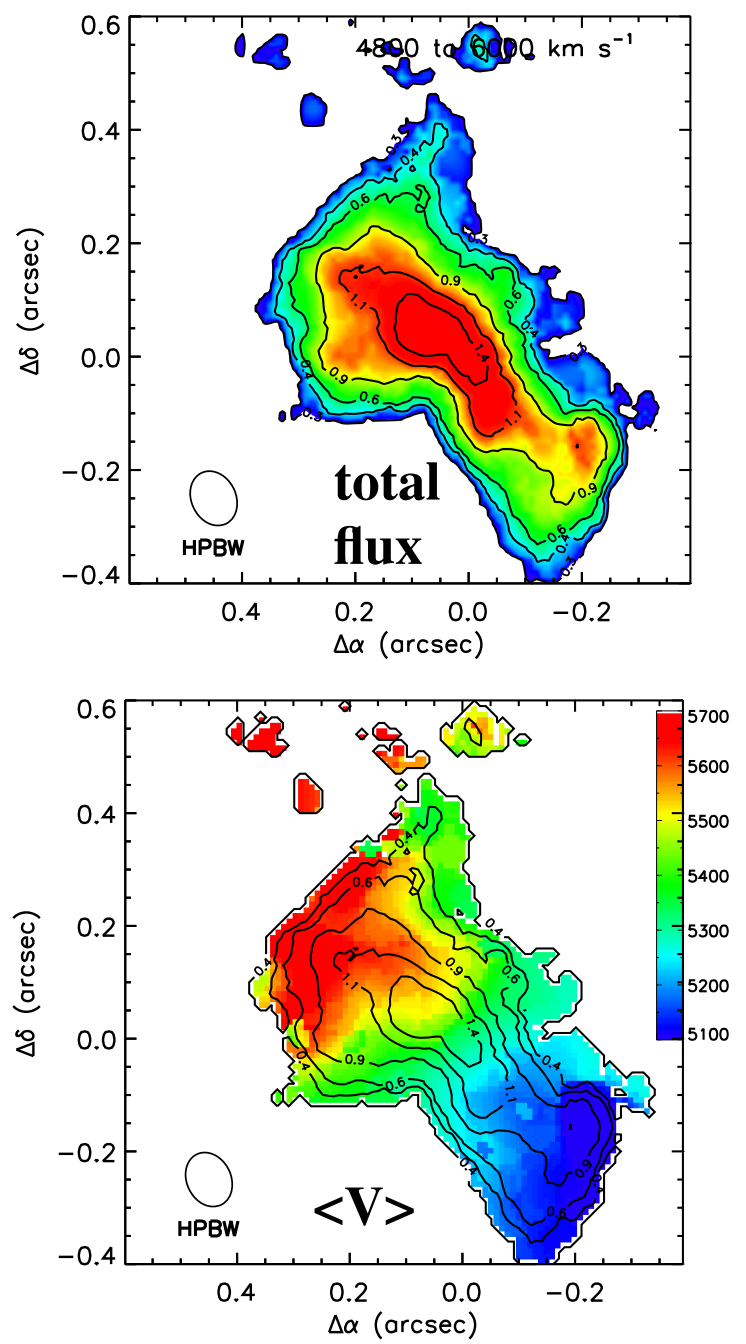

Arp 220 West CO (1-0)
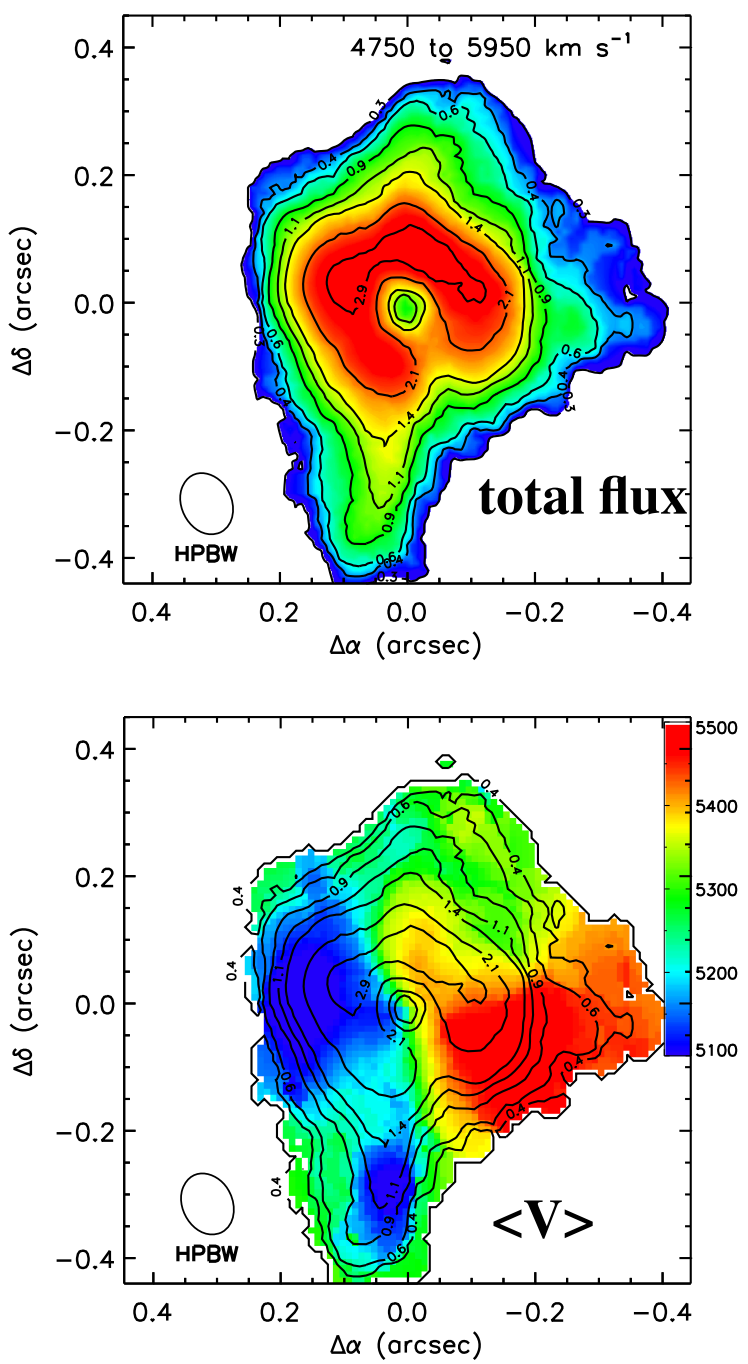

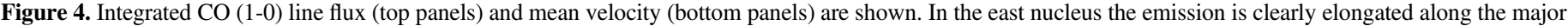

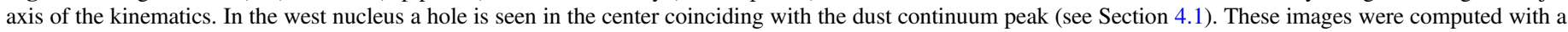

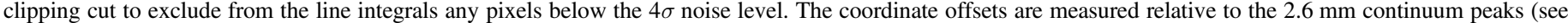
Figure 1). The contours are labeled with $\mathrm{Jy}_{\text {beam }}{ }^{-1}$ and $\mathrm{km} \mathrm{s}^{-1}$.

volume density of $n_{\mathrm{H}_{2}}=2.4 \times 10^{6} \mathrm{~cm}^{-3}$ in the central area of the west nucleus.

At these extraordinarily high ISM densities, the dust and gas will be collisionally coupled (at $n_{\mathrm{H}_{2}}>10^{4} \mathrm{~cm}^{-3}$ ) and in thermal equilibrium $\left(T_{\mathrm{D}}=T_{k}\right)$. Most molecular transitions at millimeter to submillimeter wavelengths will have level populations in thermal equilibrium with the $\mathrm{H}_{2}$ gas. And if the dust is optically thick into the millimeter regime, there will be substantial direct radiative coupling of the millimeter transitions to the dust radiation field. This is all consistent with the observed 187 K CO brightness temperature (see Table 1).

\subsection{The East Nucleus}

For the east nucleus the dust optical depth is less. Wilson et al. (2014) estimate $\tau_{434 \mu \mathrm{m}}=1.7$ and $\mathrm{T}=80 \mathrm{~K}$ (compared to the above-mentioned values of 5.6 and $181 \mathrm{~K}$ for the west nucleus). This is borne out in our $2.6 \mathrm{~mm}$ continuum imaging, which indicates a peak of just $15 \mathrm{~K}$ for the dust in the east nucleus (after removing the synchrotron and free-free contributions). This suggests $\tau_{2.6 \mathrm{~mm}} \sim 0.2$ (assuming $T_{\mathrm{D}}=80 \mathrm{~K}$ ). In this nucleus, we cannot provide the equivalent energetic constraints as in the west from our data since the dust is not optically thick at $\lambda=2.6 \mathrm{~mm}$. However, from the ratio of $\tau$ s derived by Wilson et al. (2014), one might infer that the east nucleus has $\sim 1 / 4$ the luminosity of the west nucleus. Similarly, the ratio of dust opacities in the two nuclei suggests that the mass of dust and gas in the east nucleus is $\sim 1 / 4$ of that in the west nucleus. Better constraints will be provided by the high-resolution $\mathrm{CO}(2-1)$ imaging with ALMA, which is scheduled.

\section{CO (1-0) Line Emission}

Figure 3 shows the continuum-subtracted CO (1-0) emission line profiles for the west and east nuclei obtained in apertures of 0 . 8 diameter centered on each nucleus. The $\mathrm{CO}$ emission extends up to $\sim 800 \mathrm{~km} \mathrm{~s}^{-1}$ in each of the nuclei and is offset in the mean by $\sim 120 \mathrm{~km} \mathrm{~s}^{-1}$ between the two nuclei. In the west 
nucleus the line profile exhibits broad wings and a single peak, while in the east nucleus it is double-peaked.

Images of integrated $\mathrm{CO}$ emission and the intensity-weighted centroid velocity $(\langle V\rangle)$ are shown in Figure 4 . The overall morphology of the gas distribution is remarkably different in the two nuclei. In the west nucleus, the total emission is less elongated along a major axis; there is a drop in the $\mathrm{CO}$ emission on the central resolution element (90 mas diameter); and overall, the emission is more compact (as was the case for the dust continuum).

Despite these differences, both nuclei exhibit a clear kinematic gradient, suggesting rotation. In the east nucleus, the kinematic gradient aligns closely with the major axis of the elongated $\mathrm{CO}$ intensity distribution. In the west nucleus the kinematic major axis is at $\mathrm{PA} \simeq-110^{\circ}$; in this nuclear source the emission intensity distribution is hardly elongated, and one does not see a correlation with the major kinematic axis of either the $\mathrm{CO}$ or the continuum (see Table 1). The magnitude of the mean velocity gradients is impressive; in both nuclei, the shift is $\sim 500-600 \mathrm{~km} \mathrm{~s}^{-1}$ (see Figure 4, lower panels) over 0 ". 3-0". 4 (124-165 pc).

\subsection{CO Hole on the West Nucleus}

In the west nucleus, the central dip in the $\mathrm{CO}$ emission is coincident with the central dust continuum peak. The depth of the hole is approximately a factor of 3 compared with the immediately exterior ring (see Figure 4, upper right). Since the dust continuum in the west peaks strongly in the central resolution element, this dip in the $\mathrm{CO}$ emission is probably not due to a deficiency or clearing of ISM at small radii.

There are three possible explanations for the drop in the strength of the $\mathrm{CO}$ emission at the center: (1) the $\mathrm{CO}$ and the dust are in thermal equilibrium in this high-density core; (2) the high excitation temperature of the $\mathrm{CO}$ in the core depletes the lowest $\mathrm{CO}$ rotational levels, in favor of much higher $J$ states, causing the $\mathrm{CO}(1-0)$ line to be optically thin; and (3) the CO emission from the core is self-absorbed by colder $\mathrm{CO}$ in the foreground along the line of sight.

The first explanation is consistent with the very high column density $N_{\mathrm{H} 2} \simeq 2 \times 10^{26} \quad \mathrm{H}_{2} \mathrm{~cm}^{-2}$ and volume density $n_{\mathrm{H} 2}=2.4 \times 10^{6} \mathrm{~cm}^{-3}$ deduced for the dust emission in Section 3.5. At these densities the $\mathrm{H}_{2}$ will be collisionally coupled to the grain temperature, and $T_{K}=T_{\mathrm{D}}$ and the $\mathrm{CO}$ levels will be thermalized, that is, $T_{x}=T_{K}$. In this case, no line emission will appear in excess of the optically thick dust emission. This does require that the density must drop steeply at the outer radius of the dust photosphere to avoid there being an external chromosphere producing excess $\mathrm{CO}$ line emission outside the photosphere.

The second explanation, that the CO (1-0) line in the core has lower optical depth than the dust emission, could also follow from the very high excitation conditions in the core, compared to those in normal Galactic molecular clouds. This, combined with the large velocity widths expected in the core, could cause the $\mathrm{CO}(1-0)$ to be optically thinner than the dust at $\lambda=2.6 \mathrm{~mm}$. In low-density Galactic giant molecular clouds, the $\mathrm{CO}$ (1-0) line typically has more than a factor of $10^{4}$ greater optical depth than the dust at $\lambda=2.6 \mathrm{~mm}$. In the Arp 220 nuclei, this ratio is reduced by two orders of magnitude due to the $\sim 100$ times higher gas velocity dispersion and another factor of $20-40$ due to the $\sim 20-40$ times higher gas temperature that spreads the CO molecules over more levels. In addition, the stimulated emission correction to the optical depth will cause a further reduction. A modest depletion in the $\mathrm{CO}$ abundance could then cause the (1-0) line to be optically thin.

The third explanation, having the $\mathrm{CO}$ emission from the core be self-absorbed by low-excitation $\mathrm{CO}$ farther out along the line of sight, requires that the foreground gas be coherent with that in the core, that is, at the same radial velocity. The $\mathrm{CO}$ emission at small radii will be predominantly at high velocities and thus is not coherent with gas close to the systemic velocity outside the nucleus.

In Arp 220 east, we do see three narrow absorption features (spatially offset 0 !" 1 to 0 !' 2 from the nucleus) within $\pm 100 \mathrm{~km} \mathrm{~s}^{-1}$ of the systemic velocity; these absorptions are sharp in velocity and never cover more than $\sim 30 \mathrm{~km} \mathrm{~s}^{-1}$ (e.g., Figure 3, lower left). There is negative velocity absorption seen in $\mathrm{CO}$ (3-2), $\mathrm{SiO}$ (6-5), and $\mathrm{HCO}^{+}$(3-2), which is interpreted as an outflowing wind (Sakamoto et al. 2009; GonzálezAlfonso et al. 2012; Aalto et al. 2015; Rangwala et al. 2015; Tunnard et al. 2015). However, for the wind to produce the apparent hole in CO (1-0) only from the nucleus, the wind must be confined to radii $\lesssim 16 \mathrm{pc}$. This size is inconsistent with the larger spatial extent of the high $J$ absorption lines. In addition, the outflow gas would only absorb the nuclear $\mathrm{CO}$ emission at negative velocities, leaving the other half of the emission unabsorbed.

Lastly, we note that if there were CO (1-0) absorption of the background continuum, we should expect that in the continuum-subtracted (1-0) spectra, we should see instances where the line emission has an apparent negative intensity (since too much continuum would have been subtracted at the line velocities). This is not seen in the spectra shown in Figures 3 or 9 . If the foreground $\mathrm{CO}$ was absorbing only the background line emission from the nucleus, the continuumsubtracted spectra would not necessarily go below zero, but it is not clear why the foreground absorption would not also be absorbing the nuclear continuum. The "negative" intensity $\mathrm{CO}$ line could be suppressed if the continuum and CO line emission had different spatial extents.

We thus favor the first or second explanations to reasonably account for the $\mathrm{CO}$ hole in the west nucleus. The third explanation appears inconsistent with the high spatial resolution spectra and requires overlap of radial velocities between the nucleus and the foreground gas, and if the absorption takes place in an outflowing wind, then the positive radial velocity emission would be left unabsorbed.

\subsection{CO Elongation in the East Nucleus}

In the east nucleus, the $\mathrm{CO}$ emission is clearly elongated with a major/minor axis ratio of $\sim 3: 1$ (see Figure 4, upper left) and 2:1 from the Gaussian fitting (see Table 2). If the structure is interpreted as an inclined disk, the former axis ratio implies an inclination of $70^{\circ}$ to the line of sight. The east nucleus structure is clearly not axisymmetric: the peak in the $\mathrm{CO}$ emission is displaced $\sim 0$ ". $1 \mathrm{NE}$ of the dust continuum peak (coordinate $\Delta \alpha, \Delta \delta=0,0$ in Figure 4). There is also $\sim 50 \%$ more integrated $\mathrm{CO}$ emission luminosity on the NE side than on the SW. 

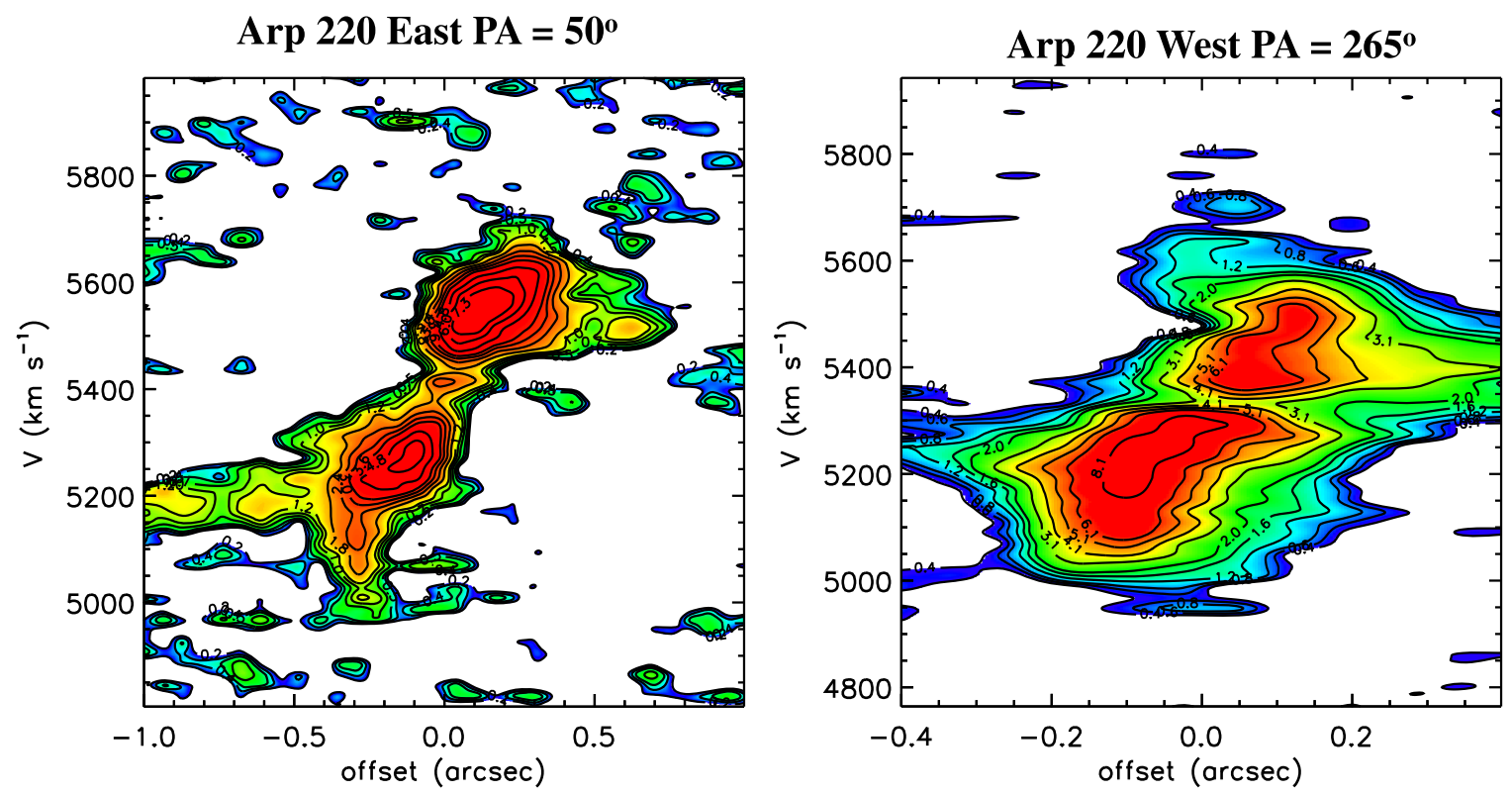

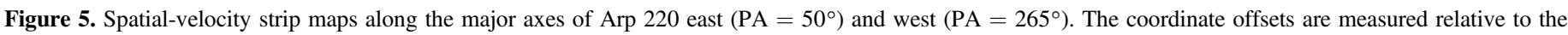

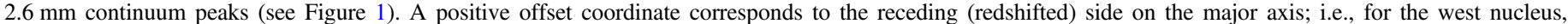

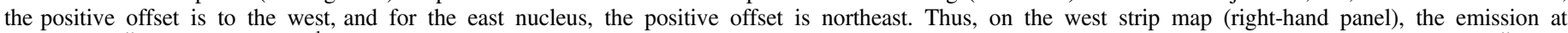

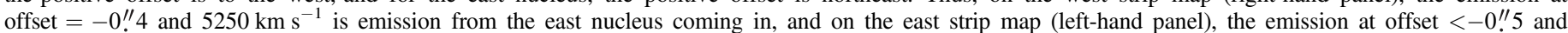
$5200 \mathrm{~km} \mathrm{~s}^{-1}$ is the west nucleus coming in. The contours are labeled with mJy beam ${ }^{-1}$.

\subsection{Major Axis Kinematics}

Figure 5 shows the distribution of $\mathrm{CO}$ emission and the gas kinematics along the major axes of the west and east nuclei. The central reference position is taken to be the $2.6 \mathrm{~mm}$ continuum peak in each nucleus. In both nuclei the velocity gradient extends over approximately $700 \mathrm{~km} \mathrm{~s}^{-1}$. In the west nucleus, this velocity range is seen within \pm 0 ". $1(R=41 \mathrm{pc})$ of the center; in the east, it occurs within \pm 0 ". 4 ( $R=165 \mathrm{pc})$ of the center. Figure 3 illustrates the contrast in spatial extent of the high-velocity emission between the two nuclei when comparing the top and bottom panels. The strip maps also clearly show the decrease in line emission on the nuclear peaks at the central velocities. At the same time, the central positions show a broad range of emission velocities: in the west, $4950-5700 \mathrm{~km} \mathrm{~s}^{-1}$, and in the east, $5150-5700 \mathrm{~km} \mathrm{~s}^{-1}$. This is also seen in the mean spectra for the nuclei shown in the Figure 3 lower panels.

\section{CO Emission Distribution and Kinematics}

In order to understand the small scale distribution of the $\mathrm{CO}$ emission and to place constraints on the gas kinematics, we have modeled each of the two nuclei with rotating disks inclined to the line of sight. This model, including a parameterized rotation curve and gas velocity dispersion, was then fit to the observed CO line profiles on a well-sampled grid (60 mas spacing) in each nucleus, in order to derive the best-fit radial distribution of $\mathrm{CO}$ line emissivity. This was carried out using the maximum likelihood procedure developed in Scoville et al. (1983) with the modification that emissivities were allowed to be different on the positive and negative offset sides of the major axis.

This modeling is done with a $2 \mathrm{D}$, infinitesimally thin planar disk with constant velocity dispersion as a function of radius.
This is obviously a much simplified model to describe the central disks in a merger system with a nearby companion and with energetic feedback from star formation and active nuclei feedback. The latter could be expected to result in velocity dispersion increasing toward the center, and the former would certainly warp any planar disk structure. However, given the limited resolution of the present observations relative to the scale of the disk, such higher order modeling is not yet warranted.

The models developed for the high-resolution CO (1-0) emission provide acceptable fits to the collection of observed CO (1-0) line profiles, with an overall reduced $\chi^{2} \sim 4-6$ for both disks. The observed and model spectra are shown in the Appendix. This is a good fit, given the simplicity of the model (biaxisymmetric with a single velocity dispersion and rotation curve). The preliminary modeling results are shown in Figure 6 and are summarized in Table 4. They establish the context for the discussion of the physical structure of the disks in Section 5.2.

The dynamical mass estimates were calculated assuming a spherical configuration (see Section 5.1). The noteworthy constraints from the disk line profile modeling are as follows: (1) a central point mass $\sim 8 \times 10^{8} M_{\odot}$ is apparently required in the west nucleus to provide a best fit to the high velocities observed in the center, but no central point mass is required for the east nucleus $\left(<10^{8} M_{\odot}\right)$; and (2) the overall dynamical masses are $\sim 1.5 \times 10^{9} M_{\odot}$ at radii less than 70 and $90 \mathrm{pc}$, respectively. We expect that these parameters may change somewhat when we simultaneously model the other $\mathrm{CO}$ transitions and include the lower resolution imaging, but they set a framework for the present models. The full modeling with the complete data sets will be presented in Scoville et al. (2016).

Two cautions are in order with respect to the possible existence of a point mass in the west nucleus. First, our modeling has 

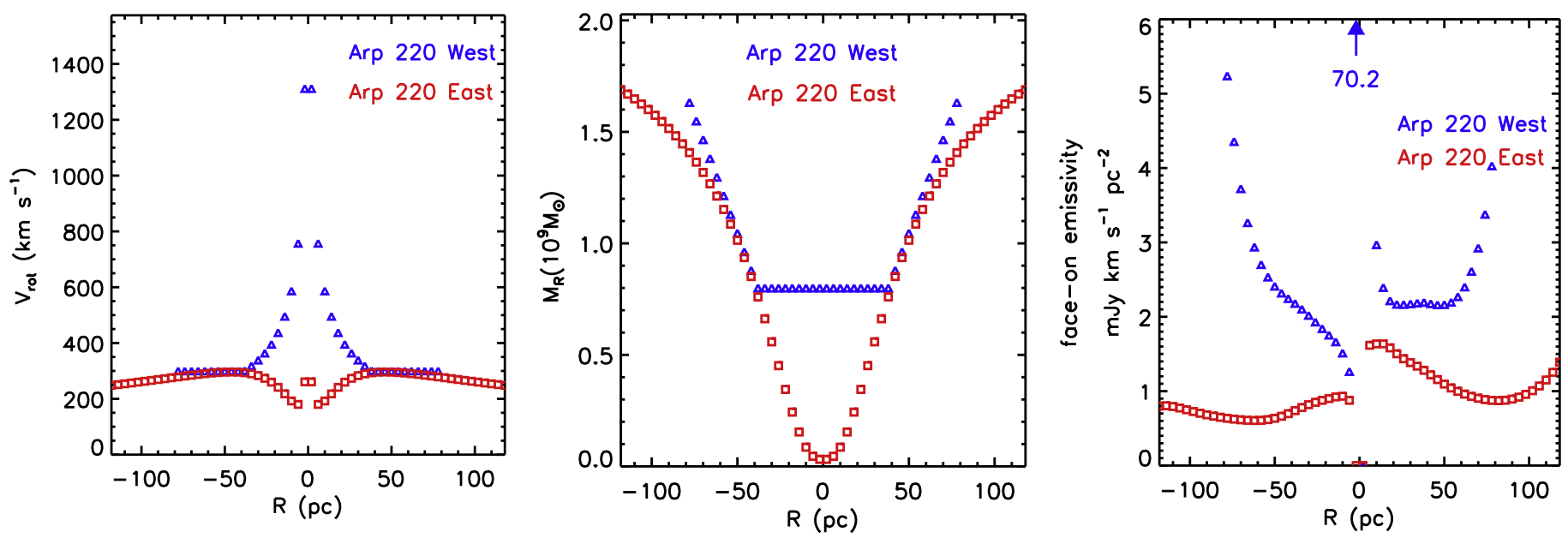

Figure 6. Derived rotation curves of enclosed dynamical mass as a function of radius are shown for the Arp 220 east and west disks as derived from fitting the highresolution line profiles (see text). The dynamical mass is obtained in the spherical approximation with $M_{R}=R V_{\mathrm{rot}}^{2} / G$. The emissivity distribution (per pc ${ }^{2}$ of area) is that which would be seen if the disks were face-on to the line of sight. The observed total integrated flux is the integral of this face-on emissivity corrected for inclination (i.e., multiplied by $\cos i$ ). A central point mass of $8 \times 10^{8} M_{\odot}$ is required in the west nucleus and only a limit of $<10^{8} M_{\odot}$ in the east nucleus. The $\mathrm{CO}(1-$ 0 ) emissivity shown in the right-hand panel is fairly flat with radius, except for the high-emissivity central point required in the west nucleus.

Table 4

Nuclear Disk Emissivity and Kinematic Models

\begin{tabular}{lll}
\hline \hline & West Nucleus & \\
\hline Systemic velocity & $V_{\text {sys }}$ & $5337 \mathrm{~km} \mathrm{~s}^{-1}$ \\
Gas turbulence & FWHM $\Delta v$ & $250 \mathrm{~km} \mathrm{~s}^{-1}$ \\
Disk inclination & $i$ & $30^{\circ}$ \\
Major axis & P.A. & $265^{\circ}$ \\
Rotation curve: & Point mass & $\sim 8 \times 10^{8} M_{\odot}$ \\
& Mass at $<70 \mathrm{pc}$ & $\sim 1.5 \times 10^{9} M_{\odot}$ \\
CO emissivity: & Peak & at $R<5 \mathrm{pc}$ \\
& Flat and $10 \times$ lower & at $R=10-50 \mathrm{pc}$ \\
& axisymmetric & \\
\hline & East Nucleus & \\
\hline Systemic velocity & $V_{\text {sys }}$ & $5431 \mathrm{~km} \mathrm{~s}^{-1}$ \\
Gas turbulence & FWHM $\Delta v$ & $120 \mathrm{~km} \mathrm{~s}^{-1}$ \\
Disk inclination & $i$ & $45^{\circ}$ \\
Major axis & P.A. & $50^{\circ}$ \\
Rotation curve: & Point mass & $<10^{8} M_{\odot}$ \\
& Mass at $<90 \mathrm{pc}$ & $1.5 \times 10^{9} M_{\odot}$ \\
CO emissivity: & 0 & at $R<5 \mathrm{pc}$ \\
& Falls a factor of 2 & out to $100 \mathrm{pc}$ \\
& Peak & at $R \sim 10 \mathrm{pc}$ \\
& Receding side $2 \times$ & \\
\hline & brighter & \\
\hline
\end{tabular}

assumed a constant velocity dispersion in each disk. This was done out of necessity to simplify the modeling. A large increase in the velocity dispersion (due to feedback) at the nucleus cannot be ruled out as a source of the large velocities at small radius. Second, the apparent point mass might be the result of extreme gas settling into the nucleus; that is, the large mass could be an interstellar gas concentration and not necessarily a black hole.

\subsection{Dynamical Masses}

Lastly, we provide a note of caution on the comparison of dynamical masses with the masses determined from the $\mathrm{CO}$ line

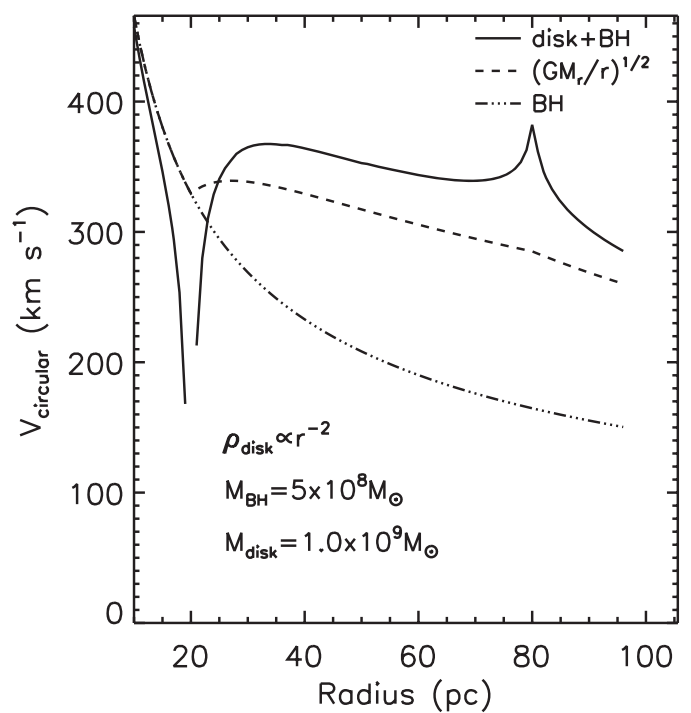

Figure 7. Circular velocities are shown for a mass distribution including a central point mass black hole (BH) of $5 \times 10^{8} M_{\odot}$ and a disk of mass $10^{9}$ $M_{\odot}$ distributed with an $r^{-2}$ density falloff between 20 and $80 \mathrm{pc}$. The solid curve shows the proper circular velocity obtained by integration over the disk mass distribution, and the dashed curve is the spherical approximation, that is, calculating $v_{\text {circ }}=\left(G M_{r} / r\right)^{1 / 2}$. The dot-dashed curve is the Keplerian rotation curve associated with the central point mass. The dip at $20 \mathrm{pc}$ is due to the net outward attraction in the inner regions of the disk; the cusp at $80 \mathrm{pc}$ is due to there being no such outward attraction disk beyond $80 \mathrm{pc}$.

and dust continuum emission. If the dynamical mass is calculated in a simple spherical approximation from $M_{\mathrm{dyn}}=r V_{\mathrm{rot}}^{2} / G$, this mass estimate may be significantly different from that calculated from the observed kinematics assuming a disk mass distribution plus a central point mass.

The mass distribution in the nuclei of Arp 220 is very likely disk-dominated, and the spherical approximation is likely to incorrectly estimate the actual mass in such a configuration. We illustrate this with a simple model (similar to Arp 220 west): a central point mass of $5 \times 10^{8} M_{\odot}$ and a disk of $10^{9} M_{\odot}$ extending between $r=20$ and $80 \mathrm{pc}$ with surface density 
varying as $r^{-2}$ and constant thickness. Figure 7 shows the circular velocity with forces determined properly for a central mass plus the disk. The circular velocity estimated in the spherical approximation (from the enclosed mass at each radius) is also shown for comparison.

The proper estimation (solid curve) and the spherical one (dashed curve) have significantly different $v_{\text {circ }}$. The differences are due to the fact that in a thin disk configuration there is significant gravitational attraction in the outward direction by gas at larger radii. We defer a proper comparison of the emission-based mass estimates to a future paper, once we have full flux recovery of the $\mathrm{CO}$ and dust emission. The rotation curve shown in Figure 7 is somewhat higher than that in Table 4, but within the range of uncertainties due to the inclination angle. In Section 6, we note an added uncertainty with the dynamical mass estimates: that radiation pressure on the dust may also provide substantial pressure support in the disks.

\subsection{Physical Structure of the Nuclear Disks}

The high-resolution imaging presented here provides strong confirmation for the existence of nuclear disk structures in both nuclei of Arp 220, as first suggested by Sakamoto et al. (1999). In the eastern nucleus the gas and dust emissions are clearly elongated, and this elongation aligns with the major axis of the $\mathrm{CO}$ velocity gradient. In Arp 220 west, the emission is not so clearly elongated, but there is a strong gradient in the $\mathrm{CO}$ line velocity at $\mathrm{PA} \simeq 265^{\circ}$. The lack of elongation in the emission distribution is likely due to the fact that the west nucleus is more compact and the kinematics suggest a more face-on inclination (inclination $i=30^{\circ}$, compared to $45^{\circ}$ in the east nucleus; see Table 4).

These nuclear disks are extraordinary structures with $\geqslant 10^{9} M_{\odot}$ within radii $<50-100 \mathrm{pc}$, and the two nuclei are only $\sim 412$ pc apart. Here, we discuss the mean ISM properties in the nuclei, the physical structure of the disks, maintenance of the disks, and the energetics of the nuclear sources. At this point, lacking the full data sets, our discussion is qualitative and intended to be illustrative of the physical considerations. The present observations do not recover all of the line and continuum flux from the region since they include only long baseline data and only the $\lambda \sim 2.6 \mathrm{~mm}$ observations; we anticipate a more thorough analysis when the low-resolution observations are completed including $\mathrm{CO}$ (2-1) and (3-2) line emissions with complete flux recovery. The observations reported in this paper do not include short spacing data, and hence they do not recover flux associated with the large-scale structures $\gtrsim 0$ ". 5 or $200 \mathrm{pc}$.

Lonsdale et al. (2006) report Very Long Baseline Interferometry (VLBI) at $18 \mathrm{~cm}$ wavelength and $\sim 1 \mathrm{pc}$ resolution, detecting 49 point-like sources, which they interpret as supernova remnants (SNRs; see also Parra et al. 2007). The sources are tightly clustered in the two nuclei, with $75 \%$ of the sources in two regions: $0.25 \times 0$ ". 15 (west nucleus) and $0.3 \times 0$ " 2 (east nucleus). In both cases these rectangles are aligned with the PA derived above for the disks. The estimated supernova (SN) rate is $4 \pm 2 \mathrm{yr}^{-1}$ based on the appearance of new SN between the epochs of the observations. The fact that 22 of the 49 SNRs are in the east nucleus and 27 in the west nucleus strongly suggests that the total star-formation rates are similar for the two nuclei, $45 \%$ versus $55 \%$. The fact that only $25 \%$ of the 49 SNR are outside the two compact nuclear regions implies that relatively little $(25 \%)$ of the total star formation occurs in the larger regions of Arp 220.

Lastly, we note one puzzle. In the west nucleus, the kinematic modeling of the intensity and velocity distributions suggests a low-inclination disk $\left(i=30^{\circ}\right.$, Table 4$)$, yet this is inconsistent with the elongated distribution of the SNRs (having a 2.5:1 major:minor axis ratio; Lonsdale et al. 2006) for which one would infer $i \simeq 60^{\circ}$ for a thin disk. Two possible resolutions of this contradiction are (1) either disk gas is more inclined than $30^{\circ}$ but the north and south extents of the west nucleus gas are increased by minor axis outflows or there is a substantial thickness to the disk or (2) the SNRs are located preferentially along one axis within a low-inclination disk. The latter might occur if the nuclear disk had a central bar in which the most recent star formation was preferentially occurring. However, the rotation period of the disk is only a few megayears, and it is unlikely that the massive stars formed in a bar would remain in a bar until the time that they undergo SN explosions. Higher resolution imaging and kinematics are probably needed to address this inconsistency.

\subsection{Summary of Observational Parameters}

For the purpose of the numerical evaluations in the discussion below, we adopt approximate estimates for the masses, radii, and luminosities for Arp 220. These are meant only for physical perspective in the discussion and are probably uncertain by factors of 2 or more. For the ISM masses, we adopt $\sim 1.5 \times 10^{9} M_{\odot}$ for each of the Arp 220 disks with their radial extents being 80 and $130 \mathrm{pc}$ for the west and east nuclear disks, respectively (Table 4), since the deconvolved radii are 74 and $11 \mathrm{pc}$ (Table 2). The velocity dispersions derived from the line profile fitting are $\sigma_{v}=\Delta v_{\mathrm{FWHM}} / 2.3 \simeq 110$ and 50 $\mathrm{km} \mathrm{s}^{-1}$, respectively $\left(\sigma_{3 \mathrm{D}}=\sqrt{3} \sigma_{1 \mathrm{D}}\right)$.

In the west nucleus the central point-like concentration has a mass of between $8 \times 10^{8}$ and $3 \times 10^{9} M_{\odot}$ as derived from the gas kinematics and the dust emission, respectively. Resolution of this discrepancy may be possible with the higher resolution $1.3 \mathrm{~mm}$ observations, which will allow better constraints on the kinematics and on the true dust temperatures in that source. Below, we adopt $10^{9} M_{\odot}$ for the unresolved mass within $R \leqslant 15$ for this extreme concentration.

The total infrared luminosity of Arp 220 is $1.9 \times 10^{12} L_{\odot}$. Using the mid-infrared, high-resolution $(\sim 0$ ". 4) photometry from Soifer et al. (1999, Table 3), we take the view that the majority of this luminosity arises from the two nuclei, apportioned $2 / 3$ (west) and $1 / 3$ (east; i.e., 1.2 and $0.6 \times 10^{12} L_{\odot}$ ). We caution that these estimates are approximate since the 3-24 $\mu \mathrm{m}$ photometry (Soifer et al. 1999) is not at the $\lambda \sim 70 \mu \mathrm{m}$ farinfrared luminosity peak; also, the distribution of SNR is more nearly equal for the two nuclei (Lonsdale et al. 2006; Parra et al. 2007). Some of the overall luminosity is also likely to originate at larger radii; the above luminosities should therefore be taken as upper limits to the luminosity of each nucleus.

\subsection{Disk Structure}

The formation of gaseous disk structures in the nuclei of merging galaxies is likely an inevitable result of the gas sinking dissipatively into the central regions more rapidly than the main stellar component of the galaxies. This results in 
a gaseous bar leading the stellar bar (since the gas has sunk to smaller radii), and the stellar bar then exerts a backward torque on the gaseous bar to further reduce its rotational angular momentum (see Barnes \& Hernquist 1992, 1996). The gas forms a rotating disk since vertical motions are efficiently damped out once the gas becomes concentrated, while the angular momentum is removed on a much longer timescale.

The thickness of the disk is determined by equilibrium between the gravitational forces toward the midplane of the disk and the gas motions in the vertical direction. These gas motions will be damped if two parcels of gas collide; their bulk kinetic energy is then converted to thermal energy in shocks. The high density of the molecular gas ensures that the shocks radiate the postshock thermal energy very efficiently. The net result is that the kinetic energy associated with vertical motions needed to maintain the disk thickness is radiated on a timescale similar to the collision time of the gas parcels. In order to maintain the thickness of the disk and its associated vertical motions on a longer timescale, constant replenishment of the turbulent energy is required.

The typical timescale for collision of gas parcels and the dissipation of the turbulent motions is given by the vertical crossing time of the disk and the fraction of the disk area filled by gas parcels as viewed perpendicular to the disk:

$$
\begin{aligned}
\tau_{\text {dis }} & =\tau_{\text {cross }} / f_{a} \\
& =H /\left(\sigma_{v} f_{a}\right) .
\end{aligned}
$$

Here $H$ is the full thickness of the disk, and $f_{a}$ is the areal covering factor of the disk.

\subsection{Disk Area Covering}

The disk areal covering factor must be of order unity. The observed CO peak brightness temperature is comparable with the estimated dust brightness temperatures $(200 \mathrm{~K})$ and hence the dust temperature, since the dust is nearly optically thick. The $\mathrm{CO}$ peak brightness temperature in the west disk is $187 \mathrm{~K}$ (Table 1), and the areal covering factor for the CO-emitting gas must be $\sim 1$. In the east disk, the $\mathrm{CO}$ peak is $175 \mathrm{~K}$, implying a similarly high areal covering factor.

\subsection{Disk Thickness}

For the nuclear disks, we may estimate the disk thickness assuming that the vertical distribution is determined by equilibrium between the gravitational force in the $z$ direction and the observed gas $1 \mathrm{D}$ velocity dispersions $\left(\sigma_{v}(1 \mathrm{D})\right)$. We do this under the very simple assumption that the vertical velocity dispersion is constant with radius and that the disks are in equilibrium. Specifically, this assumes that outflow winds are not contributing to the vertical scale height.

We consider two cases: (1) where the gas disk mass surface density is much less than that of the stellar disk or spheroid, and (2) where the surface density is dominated by the gaseous disk (i.e., a fully self-gravitating disk). The former is appropriate for low- $z$ galactic disks, where the ISM mass is typically only $5 \%-10 \%$ of the stellar mass. The latter is likely to be most appropriate for high- $z$ galaxies with large gas-mass fractions or the gaseous nuclear disks as in Arp 220 where the gas has been preferentially funneled to the nucleus faster than the stars.

In the non-self-gravitating case, the vertical distribution of the gas will have density $\rho=\rho_{0} \exp \left(-z^{2} / z_{0}^{2}\right)$. If we define the disk thickness $(H)$ as the full thickness at which the density has dropped by a factor $1 / e$, then $H=2 z_{0}$. For a spherical distribution of stars,

$$
H(r)=2 \sqrt{2}\left(\frac{\sigma_{v}(1 \mathrm{D})}{V_{\mathrm{rot}}}\right) r
$$

For a typical low- $z$ galaxy where the stellar distribution is disklike, the vertical frequency $\nu_{z} / \Omega_{\text {rot }} \sim 3$, and the thickness is reduced by a corresponding factor of $\sim 3$.

In a fully self-gravitating gas disk, the vertical distribution is $\rho=\rho_{0} \operatorname{sech}^{2}\left(z /\left(2 z_{0}\right)\right)$ with

$$
\begin{aligned}
z_{0}(r) & =\frac{\sigma_{v}(1 \mathrm{D})}{\sqrt{8 \pi G \rho_{0}}} \\
& =\frac{\sigma_{v}(1 D)^{2}}{2 \pi G \Sigma_{\mathrm{disk}}},
\end{aligned}
$$

where $\Sigma_{\text {disk }}$ is the mass surface density of the gas disk. For a Mestel disk, $\Sigma_{\text {disk }}=V_{\text {rot }}^{2} /(2 \pi G r)$, and therefore

$$
z_{0}(r)=\left(\frac{\sigma_{v}(1 \mathrm{D})}{V_{\mathrm{rot}}}\right)^{2} r
$$

In this case, the equivalent full thickness at which the density has dropped by $1 / e$ is $H(r) \simeq 4.34 z_{0}$ :

$$
H(r)=4.34\left(\frac{\sigma_{v}(1 \mathrm{D})}{V_{\mathrm{rot}}}\right)^{2} r
$$

Figure 8 shows the disk scale thickness $(H)$ variation as a function of $r$ for the west and east disks (Table 4), indicating $H$ ranging from 1 to $30 \mathrm{pc}$ at $r<50 \mathrm{pc}$. The disk crossing times (and thus the turbulent dissipation timescales) shown in the right-hand panel of Figure 8 are $10^{5}$ to $4 \times 10^{5}$ years.

In order to maintain the disk vertical structure and its observed velocity dispersion, it is required that the turbulent energy in the gas motions be replenished on a similar timescale. The sources of this input might include (1) starburst and AGN power and the momentum associated with their radiation, (2) the pressure support provided by radiation liberated by the turbulence dissipation, and (3) the gravitational potential energy released as the gas accretes inward and the potential energy associated with the decaying orbits of the two nuclei. These are discussed and evaluated in Section 5.8.

\subsection{Disk Gas Properties}

For the masses and radii given above, we estimate the mean gas densities in the two disks. From the fitting of the line profiles across the two disks, the best-fit model has approximately constant $\mathrm{CO}$ line emissivities as a function of radius outside the central $R=10 \mathrm{pc}$, with the west disk having a factor of 2-3 higher mean emissivity compared to the east disk; 

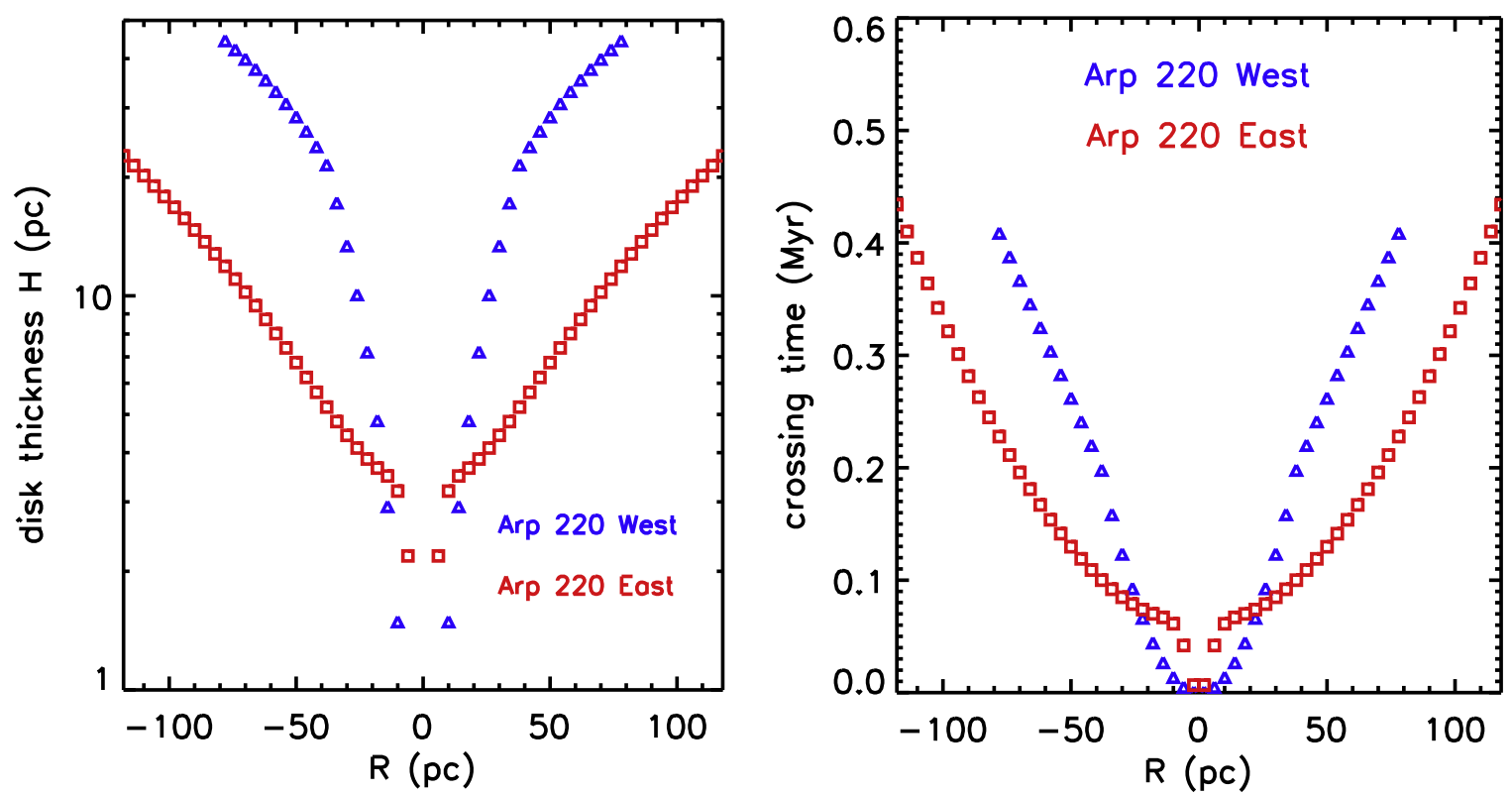

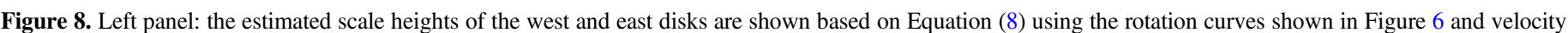

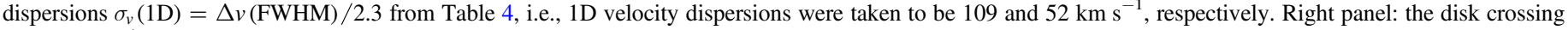
timescale $\left(\mathrm{H} / \sigma_{v}(1 \mathrm{D})\right)$, which is equivalent to the turbulent dissipation timescales calculated from Equation (4) with $f_{a}=1$.

on the other hand, the east disk has a larger radial extent. We adopt a mean disk thickness in the $z$ direction of $10 \mathrm{pc}$. The mean $\mathrm{H}_{2}$ density (not correcting for $\mathrm{He}$ ) is then given by

$$
\begin{aligned}
\left\langle n_{\mathrm{H}_{2}}\right\rangle= & M_{\text {disk }} /\left(4 \pi \mathrm{R}^{2} \mathrm{H} \mathrm{m}_{\mathrm{H}_{2}}\right) \\
\left\langle n_{\mathrm{H}_{2}}\right\rangle= & 1.7 \times 10^{4}\left(\frac{M_{\text {disk }}}{10^{9} M_{\odot}}\right) \\
& \times\left(\frac{10 \mathrm{pc}}{H_{\mathrm{FWHM}}}\right)\left(\frac{100 \mathrm{pc}}{R_{\text {disk }}}\right)^{2} \mathrm{~cm}^{-3} .
\end{aligned}
$$

For the mean thickness of $10 \mathrm{pc}$ and density $n_{\mathrm{H}_{2}} \simeq$ $1.7 \times 10^{4} \mathrm{~cm}^{-3}$, the column density through the disk is $N_{\mathrm{H}_{2}}=5.2 \times 10^{23} \mathrm{~cm}^{-2}$. Assuming a standard Galactic ISM gas-to-dust ratio $N_{\mathrm{H}_{2}} / A_{V}=1.0 \times 10^{21} \mathrm{~cm}^{-2} \mathrm{mag}^{-1}$, we find a typical extinction of $A_{V}=520 \mathrm{mag}$. The disks will thus be optically thick to any NIR-UV radiation emitted by young stars formed within the disks.

One final question is the structure of the molecular gas disk: Is it composed of a smooth gas distribution or discrete clouds (e.g., giant molecular clouds, or smaller clouds)? If the observed velocity dispersion $\sigma=50-100 \mathrm{~km} \mathrm{~s}^{-1}$ is due to internal motions within individual clouds and if these clouds are gravitationally bound, virialized, and have uniform density, then their masses are $M_{\text {cloud }}=5 / 3 R \sigma_{v}$ (internal) $)^{2} / G$. If the 3D $\sigma_{v}$ (internal $)=100 \mathrm{~km} \mathrm{~s}^{-1}$ and one requires that their density be twice the mean disk density $\left(\left\langle n_{\mathrm{H}_{2}}\right\rangle=1.7 \times 10^{4} \mathrm{~cm}^{-3}\right)$, their masses must be $\sim 7 \times 10^{7} M_{\odot}$ with radii $=18 \mathrm{pc}$ (the resolution of the observations). However, the area covering factor for the disk would then be inadequate $\left(f_{a} \sim 0.1\right)$, and their size would be larger than the disk thickness.

For higher density self-gravitating clouds, the area covering factor is even lower. Lower density self-gravitating clouds would be larger and have a more appropriate area covering factor, but then their density contrast would be sufficiently small that one might as well think of the disks as continuous rather than as a cloudy medium. We are thus led to the conclusion that the gas cannot be in discrete clouds but must be a fairly continuous medium (albeit with some clumping) distributed over the disk area.

To summarize, (1) if the gas is indeed relatively smooth (i.e., not in discrete clouds as in the Galactic disk), it must still have highly supersonic motions with Mach number $>100$. (2) If these motions are not in the form of smoothly varying flows, then the turbulent kinetic energy dissipates on a disk crossing time of $\sim 10^{5}$ years (Figure 8 , right). (3) The turbulent kinetic energy must then be replenished within the dissipation timescale in order for the structure to last at least one rotation period of the disk. If the disk does not last this minimum time period, we should not expect to see a disk structure with the high velocity dispersion observed. (4) Alternatively, if the motions are in an ordered flow (and therefore less dissipative), the scale over which the velocity field varies must be smaller than the spatial resolution of our observations of $\sim 30 \mathrm{pc}$.

A hybrid picture-clouds of dense gas (with low areal covering factor) embedded in a diffuse molecular medium of low column but high enough areal covering factor to account for the $\mathrm{CO}$ brightness temperatures - might also be viable. Most of the mass would have to be in the compact, high-density clouds to avoid having the kinetic energy associated with their bulk motion rapidly dissipated by friction as they move through the diffuse medium. In this case, a large reservoir of kinetic energy is stored in the compact clouds, which stir up motions in the diffuse medium without dissipating significant kinetic energy. And of course, such clumps might not be long-lived, but rather are being formed and then destroyed. Discriminating this hybrid picture from a more continuous model will require highresolution measurements of the brightness temperatures for high excitation emission lines, to assess the areal covering factor of 
the dense component, and rare isotopes of $\mathrm{CO}$ to constrain the line optical depths.

\subsection{Energy and Momentum Considerations}

There are several sources of energy input to the gas that might replenish whatever losses there are in shock fronts: the radiative and mechanical energy and momentum input from the starburst, the gravitational potential energy resulting from shrinking of the two disks, and the final merging of the galactic nuclei. Taking a characteristic timescale of $1 \mathrm{Myr}$ (which is an upper limit to the dissipation timescale if the motions are dissipative), we may compare these sources.

The total energy in the radiation field associated with $L=10^{12} L_{\odot}$ integrated over $1 \mathrm{Myr}$ is $1.1 \times 10^{59} \mathrm{erg}$. The total energy contained in the motions associated with the observed velocity dispersion $\sigma_{v}(1 \mathrm{D})=100 \mathrm{~km} \mathrm{~s}^{-1}$ is $3 / 2\left(M \sigma_{v}(1 \mathrm{D})^{2}\right)=$ $4.5 \times 10^{56} \mathrm{erg}$ for $M_{\text {ISM }}=1.5 \times 10^{9} M_{\odot}$ (the 3 is to convert to a $3 \mathrm{D}$ velocity dispersion). The potential energy associated with each disk and with the binary merging galactic nuclei is larger than that calculated above for the dispersive gas motions, by a factor $\left.V / \sigma_{v}(3 \mathrm{D})\right)^{2} \gtrsim 1$, where $V$ is the disk rotation velocity or the relative velocity of the nuclei.

Although the energy in the radiation field is vastly larger than that in the gas motions, it is more appropriate to compare the radiation momentum with that in the gas, since the gas motions are more likely to be driven by the radiation pressure than by expansion of hot gas heated by the radiation field.

The radiative momentum input ( $L / c$ per unit time) integrated over $1 \mathrm{Myr}$ is $3.7 \times 10^{48} \mathrm{grcm} \mathrm{s}^{-1}$; the momentum in the gas is $3 \times 10^{49} \mathrm{gr} \mathrm{cm} \mathrm{s}^{-1}$, that is, an order of magnitude larger. The momentum input over the same time period from supernovae at a rate of $\sim 4 \mathrm{yr}^{-1}$ (Lonsdale et al. 2006; assuming $5 M_{\odot}$ of ejecta in the momentum-conserving phase starting at $v_{\text {exp }} \lesssim 1000 \mathrm{~km} \mathrm{~s}^{-1}$ ) is $\lesssim 4 \times 10^{48} \mathrm{gr} \mathrm{cm} \mathrm{s}^{-1}$. In summary, if the gas motions are dissipated on a disk crossing timescale, there is no obvious source for their replenishment other than the large-scale gravitational field of the galaxy.

\subsection{A Semicoherent Disk Wave Pattern}

In the last section, we found that there are no known localized sources of energy to replace that which is dissipated on a disk crossing timescale. Here we explore the possibility that the observed velocity dispersion is due to "coherent" flows or waves within the disks. The flows must be semicoherent to reduce dissipation. The scale over which these flows must span the full range of velocities $\left(\simeq 2 \sigma_{v}\right)$ must be significantly smaller than the observational resolution of 37 pc. Clearly, this will not avoid all dissipation, since there will be some regions where the flow still has shocks, but in the absence of dispersive motions throughout, the overall dissipation might be reduced an order of magnitude. One possible scenario could be wave motions in the disks generated by the tidal field of the two merging galaxy nuclei-a dumbbell-like potential. The characteristic scale of such gravitational perturbations is $\gtrsim 100 \mathrm{pc}$, that is, much larger than the required $30 \mathrm{pc}$ scale. On the other hand, one expects that the energy input at long wavelengths should decay to smaller wavelengths, as in a turbulent cascade.
A gas parcel of unit mass moving in the $z$ direction perpendicular to the disk will execute a harmonic motion relative to the midplane with the restoring force due to the central point mass and the disk mass at lower $z$ height:

$$
\begin{aligned}
F_{\text {central }} & =-G \frac{M_{r}}{\left(r^{2}+z^{2}\right)^{3 / 2}} z \simeq-G \frac{M_{r}}{r^{3}} z \text { and } \\
F_{\text {disk }} & =-4 \pi G \rho z
\end{aligned}
$$

where the disk is assumed to have constant mass density $\rho$ over scale height $H$ at radius $r$, and $M_{r}$ is the mass interior to $r$ (disk and central mass). The scale length $H$ for the harmonic motion is then

$$
\begin{aligned}
H^{2} & =\frac{2 \sigma_{v}^{2}}{\omega_{c}^{2}+\omega_{d}^{2}} \text { where } \\
\omega_{c}^{2} & =\frac{G M_{r}}{r^{3}} \text { and } \quad \omega_{d}^{2}=4 \pi \mathrm{G} \rho .
\end{aligned}
$$

These waves would be analogous to gravity waves, possibly generated by the tightly wrapped spiral pattern excited in latestage mergers by the companion nucleus.

\section{Radiation Pressure Support}

As noted in Section 5.1, it might appear difficult to reconcile the mass estimates for ISM within the disks (as well as any central components such as a black hole or compact dust source) with the observed rotation curves assuming circular motions. However, the high opacity of the dust will mean that there is substantial absorption and re-emission of the infrared radiation and, therefore, pressure support from the radiation field, since the dust is optically thick, even at far-infrared wavelengths (Scoville 2003; Thompson et al. 2005, 2015).

Assuming the Mestel disk, with $L_{r}$ and $M_{r}$ being the luminosity and mass as a function of radius in the disk, then the condition that inward gravitational forces are balanced by the outward radiation pressure is

$$
\begin{aligned}
\frac{L_{r} \kappa \rho}{c 2 \pi r H} & \simeq \frac{G M_{r} \rho}{r^{2}}, \text { implying } \\
\frac{L_{r}}{M_{r}} & =\frac{c 2 \pi H G}{r \kappa},
\end{aligned}
$$

where $\kappa$ is the radiation absorption coefficient per unit mass weighted to the effective wavelength of the infrared radiation field at each radius. Using Equation (8) for the ratio $H / r$, Equation (11) becomes

$$
\frac{L_{r}}{M_{r}}=\frac{8.6 \pi \mathrm{c}}{\kappa} G\left(\frac{\sigma_{v}}{V_{\mathrm{rot}}}\right)^{2} .
$$

Lastly, we require $\kappa$ at the wavelengths of the far-infrared radiation in the disks. For the standard ISM gas-to-dust ratio $N_{\mathrm{H}} / A_{V}=2 \times 10^{21} \mathrm{~cm}^{-2}, \kappa_{V} \simeq 312 \mathrm{~cm}^{2} \mathrm{gr}^{-1}$, and for $\kappa_{\mathrm{IR}} / \kappa_{V} \sim 1 / 30$ (Pollack et al. 1994; Scoville 2013; Thompson 
et al. 2015), $\kappa_{\mathrm{IR}} \simeq 10 \mathrm{~cm}^{2} \mathrm{gr}^{-1}$. Equation (12) then becomes

$$
\frac{L_{r}}{M_{r}}=312\left(\frac{\sigma_{v} / V_{\mathrm{rot}}}{1 / 3}\right)^{2} \frac{L_{\odot}}{M_{\odot}} .
$$

For a disk of luminosity of $\sim 5 \times 10^{11} L_{\odot}$ and mass of $\sim 10^{9}$ $M_{\odot}$ as in Arp 220, the luminosity-to-mass ratio is $500 L_{\odot} / M_{\odot}$. It is therefore clear that radiation on the dust in the central regions of Arp 220 is likely to be very important in providing significant pressure support against gravity. Of course this also implies that estimates of the dynamical mass from the observed kinematics may substantially underestimate the enclosed masses. In this case, the radiation is providing significant pressure support, and thus the required circular velocities are smaller.

The analysis above did not allow for the higher escape probability of the IR radiation in the $z$ direction (as opposed to the radial direction). However, the disks are also quite optically thick in the $z$ direction at the wavelengths of the $\sim 100-200 \mathrm{~K}$ radiation field, so the escape probability perpendicular to the disks is low.

\section{Summary Discussion}

These CO (1-0) and dust continuum observations with ALMA at 90 mas resolution clearly resolve the two nuclei in Arp 220. The two nuclei have rotating disks of radii 74 (west) and $111 \mathrm{pc}$ (east; Table 2). The west nucleus also has a massive, unresolved dust emission source in its center that is optically thick at $\lambda=2.6 \mathrm{~mm}$; the column density of gas in this compact source is $\sim 2 \times 10^{26} \mathrm{~cm}^{-2}$, assuming the dust has normal ISM dust opacity and abundance. This column corresponds to an incredible $A_{V}=2 \times 10^{5} \mathrm{mag}$ and $900 \mathrm{gr} \mathrm{cm}^{-2}$, equivalent to a $1 \mathrm{ft}$ thick wall of gold!

Modeling the observed CO emission with rotating 2D disks in each nucleus yields acceptable fits to the observed line profiles. The kinematics of the west nucleus suggest a central point mass of $\sim 8 \times 10^{8} M_{\odot}$ (an extremely compact gas concentration of a central black hole). Typical velocity dispersions in the gas are $\sigma_{v} / V_{\text {rot }} \sim 3$, indicating disk thicknesses between 1 and $30 \mathrm{pc}$ over the range of radii. The calculated timescales for dissipation of the motions represented in the velocity dispersion are $\sim 10^{5}$ years if these motions are turbulent. Since there does not appear to be a source for renewal of the turbulent energy at this short timescale, we suggest that there may be coherent wave-like motions in the disks. Such motions might be associated with the rapid wrapping of a spiral pattern generated by the close proximity of the nuclei.

One of the most impressive aspects of the structure in the nuclei of Arp 220 is its symmetry and regularity. Despite the fact that this is a very late stage galaxy merger with nuclei only $400 \mathrm{pc}$ apart, the two disks are symmetric to a factor of 2 in brightness, and their kinematics can be modeled reasonably well with axisymmetric rotation curves. This symmetry likely implies that each disk has rotated several revolutions within the time over which they significantly change their separation. That is, they have had time to relax during the merging process. For both disks, the rotation velocity is $300 \mathrm{~km} \mathrm{~s}^{-1}$ at $50 \mathrm{pc}$ radius, so this rotation period is $1 \mathrm{Myr}$; the observed symmetry therefore implies a merging timescale of $\gtrsim 3-5$ Myr.

The above is not to say there are no asymmetries. On the west nucleus, there is a deficiency in the emission to the SW of the nucleus, that is, a break in the ring of peak emission encircling the core. In addition, there is also elongation of blueshifted emission to the south of the nucleus (see Figure 4). And in the east nucleus, the line emission peak is displaced $\sim 0$ " $07 \mathrm{NE}$ of the continuum peak $(\Delta \alpha, \Delta \delta=0,0$ in Figure 4).

To put the Arp 220 structures in perspective, it is useful to compare with the dense gas structures in the center of the Milky Way. There the most massive gas concentrations are the Sgr B2 and Sgr A molecular clouds (each several $10^{6} M_{\odot}$ and size $\sim 30 \mathrm{pc}$ ), which are separated by over $150 \mathrm{pc}$. The total molecular gas mass inside a $300 \mathrm{pc}$ Galactic radius is $\sim 10^{8} \mathrm{M}_{\odot}$; thus only a small fraction of the area in the nuclear disk of the Milky Way is covered by dense gas, in contrast to Arp 220, where the areal filling factors are $\sim 1$.

There are several topics we have not discussed here since they are more appropriately treated once we have the full $\mathrm{CO}$ (2-1) and (3-2) data sets with both high- and low-resolution imaging for full flux recovery. These include a comprehensive assessment and comparison of the masses (derived from line and dust emission and from the kinematics); the environs and interaction of the two nuclei; and an excitation analysis to estimate abundances, densities, and temperatures in the gas. All are deferred to our later paper.

We thank the referee for a very thorough review of the manuscript and useful suggestions for clarification. We thank Zara Scoville for proofreading the manuscript.

This paper makes use of the following ALMA data: https: //almascience:nrao:edu/aq/?project code = 2015:1:00113:S. ALMA is a partnership of ESO (representing its member states), NSF (USA), and NINS (Japan), together with NRC (Canada) and NSC and ASIAA (Taiwan), in cooperation with the Republic of Chile. The Joint ALMA Observatory is operated by ESO, AUI/NRAO, and NAOJ. This work was done in part at the Aspen Center for Physics, which is supported by National Science Foundation grant PHY -1066293. T.A.T. is supported by NSF Grant \#1516967. T.A.T. thanks the Simons Foundation and organizers Juna Kollmeier and Andrew Benson for support for the Galactic Winds: Beyond Phenomenology symposium series.

\section{Appendix Observed and Model-fit CO Spectra}

In Figures 9 and 10 we show the observed and model $\mathrm{CO}$ spectra sampled on a 60 mas grid for the west and east nuclei. The spectra are shown here so that the reader can see that even at 90 mas resolution the $\mathrm{CO}$ emission has a large velocity dispersion $\sigma_{v}=110$ and $50 \mathrm{~km} \mathrm{~s}^{-1}$ for the west and east nuclei. The appropriateness of the model-fit parameters (see Section 5) can be judged by comparison of the observed (blue) and model (red) spectra. The model was a very simple semiaxisymmetric emissivity distribution with a parameterized rotation curve and a single velocity dispersion. 

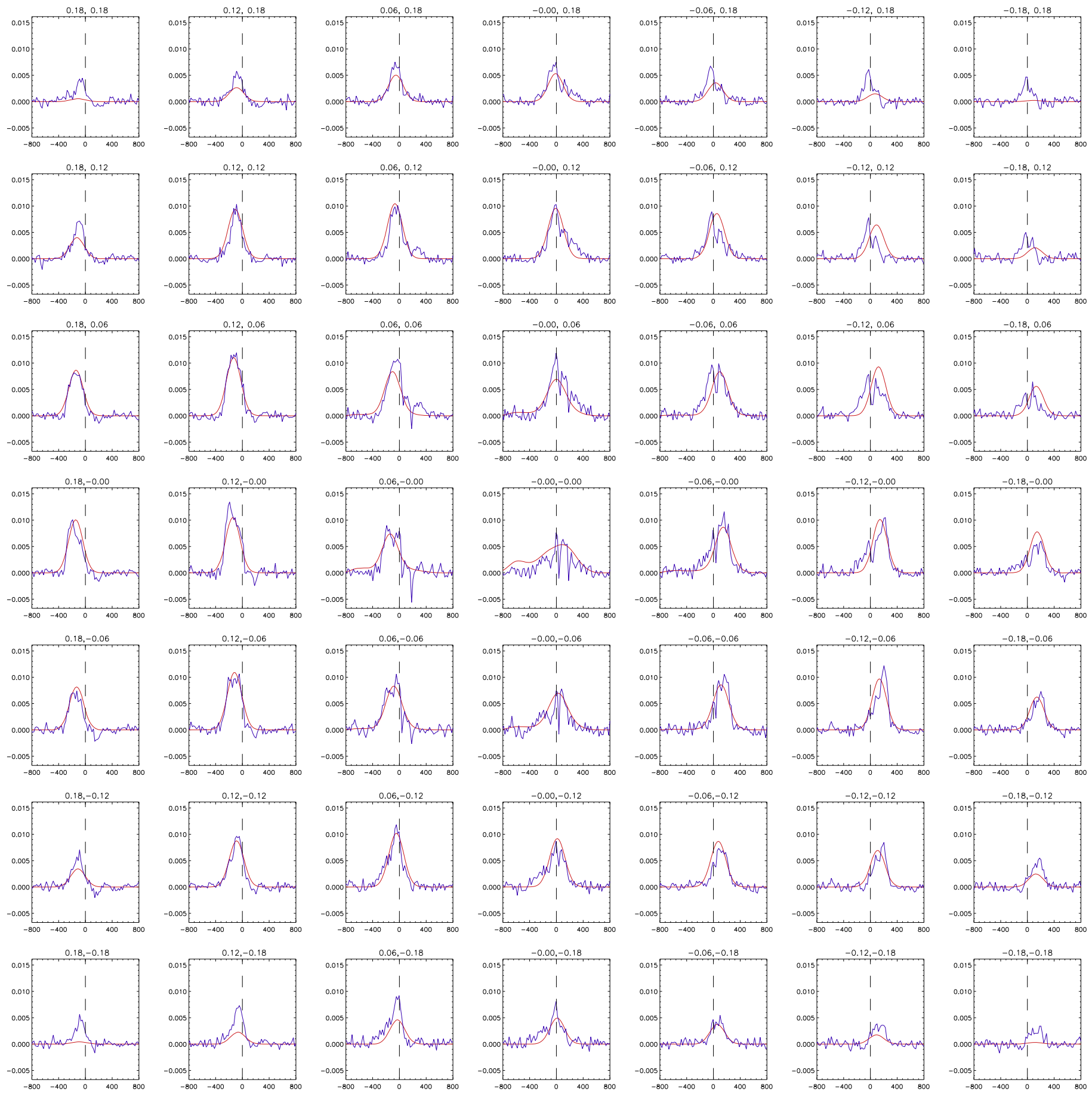

Figure 9. Observed and model spectra (blue and red curves, respectively) are shown for a 60 mas grid centered on Arp 220 west. The legend above each spectrum provides the angular offset in arcsec, and the velocity scale is relative to the west nucleus systemic velocity $v_{\text {radio }}=5337 \mathrm{~km} \mathrm{~s}^{-1}$. 

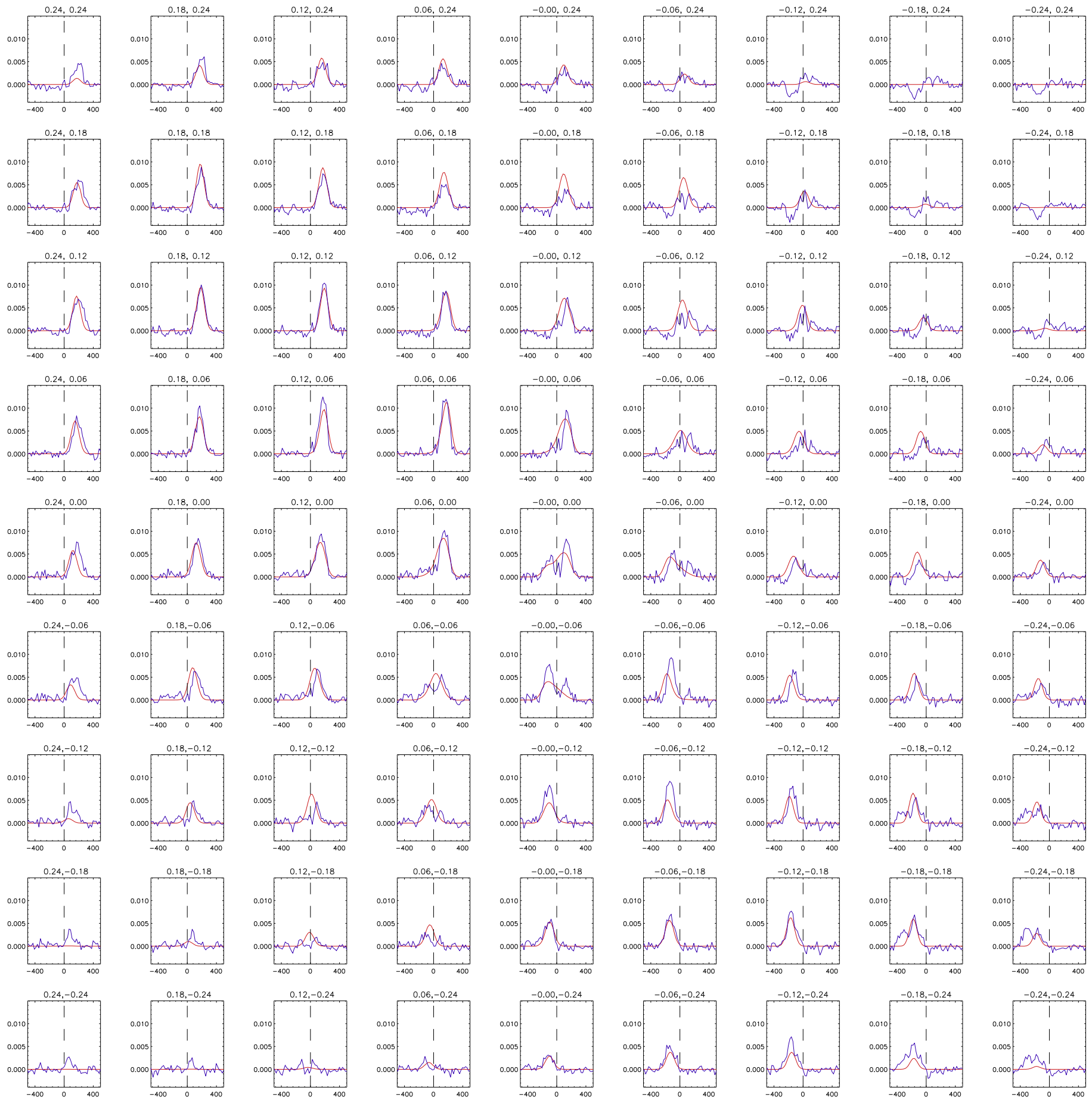

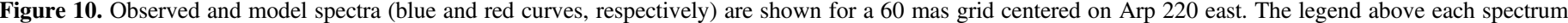
provides the angular offset in arcsec, and the velocity scale is relative to the east nucleus systemic velocity $\mathrm{v}_{\text {radio }}=5431 \mathrm{~km} \mathrm{~s}^{-1}$.

\section{References}

Aalto, S., Martin, S., Costagliola, F., et al. 2015, A\&A, 584, A42

Armus, L., Heckman, T., \& Miley, G. 1987, AJ, 94, 831

Armus, L., Mazzarella, J. M., Evans, A. S., et al. 2009, PASP, 121, 559

Barcos-Muñoz, L., Leroy, A. K., Evans, A. S., et al. 2015, ApJ, 799, 10

Barnes, J. E., \& Hernquist, L. 1992, ARA\&A, 30, 705

Barnes, J. E., \& Hernquist, L. 1996, ApJ, 471, 115

Caputi, K. I., Lagache, G., Yan, L., et al. 2007, ApJ, 660, 97

Downes, D., \& Eckart, A. 2007, A\&A, 468, L57

González-Alfonso, E., Fischer, J., Graciá-Carpio, J., et al. 2012, A\&A, 541, A4

Greve, T. R., Papadopoulos, P. P., Gao, Y., \& Radford, S. J. E. 2009, ApJ, 692, 1432

Le Floc'h, E., Aussel, H., Ilbert, O., et al. 2009, ApJ, 703, 222
Lonsdale, C. J., Diamond, P. J., Thrall, H., Smith, H. E., \& Lonsdale, C. J. 2006, ApJ, 647, 185

Magnelli, B., Popesso, P., Berta, S., et al. 2013, A\&A, 553, A132

Martin, S., Aalto, S., Sakamoto, K., et al. 2016, A\&A, 590, A25

Matsushita, S., Iono, D., Petitpas, G. R., et al. 2009, ApJ, 693, 56

Parra, R., Conway, J. E., Diamond, P. J., et al. 2007, ApJ, 659, 314

Planck Collaboration 2011a, A\&A, 536, A21

Planck Collaboration 2011b, A\&A, 536, A25

Pollack, J. B., Hollenbach, D., Beckwith, S., et al. 1994, ApJ, 421, 615

Rangwala, N., Maloney, P. R., Glenn, J., et al. 2011, ApJ, 743, 94

Rangwala, N., Maloney, P. R., Wilson, C. D., et al. 2015, ApJ, 806, 17

Rodriguez-Gomez, V., Genel, S., Vogelsberger, M., et al. 2015, MNRAS, 449,49

Sakamoto, K., Aalto, S., Wilner, D. J., et al. 2009, ApJL, 700, L104 
Sakamoto, K., Scoville, N. Z., Yun, M. S., et al. 1999, ApJ, 514, 68

Sakamoto, K., Wang, J., Wiedner, M. C., et al. 2008, ApJ, 684, 957

Sanders, D. B., Mazzarella, J. M., Kim, D.-C., Surace, J. A., \& Soifer, B. T. 2003, AJ, 126, 1607

Sanders, D. B., \& Mirabel, I. F. 1996, ARA\&A, 34, 749

Sanders, D. B., Scoville, N. Z., \& Soifer, B. T. 1991, ApJ, 370, 158

Sanders, D. B., Soifer, B. T., Elias, J. H., et al. 1988, ApJ, 325, 74

Scoville, N. 2003, JKAS, 36, 167

Scoville, N. 2013, arXiv:1210.6990v2

Scoville, N., Sheth, K., Aussel, H., et al. 2015a, arXiv:1511.05149

Scoville, N., Sheth, K., Aussel, H., et al. 2016, arXiv:1605.099381

Scoville, N., Sheth, K., Walter, F., et al. 2015b, ApJ, 800, 70
Scoville, N. Z., Evans, A. S., Dinshaw, N., et al. 1998, ApJL, 492, L107

Scoville, N. Z., Young, J. S., \& Lucy, L. B. 1983, ApJ, 270, 443

Scoville, N. Z., Yun, M. S., \& Bryant, P. M. 1997, ApJ, 484, 702

Soifer, B. T., Neugebauer, G., Matthews, K., et al. 1999, ApJ, 513, 207

Solomon, P. M., Downes, D., Radford, S. J. E., \& Barrett, J. W. 1997, ApJ, 478, 144

Teng, S. H., Rigby, J. R., Stern, D., et al. 2015, ApJ, 814, 56

Thompson, T. A., Fabian, A. C., Quataert, E., \& Murray, N. 2015, MNRAS, 449, 147

Thompson, T. A., Quataert, E., \& Murray, N. 2005, ApJ, 630, 167

Tunnard, R., Greve, T. R., Garcia-Burillo, S., et al. 2015, ApJ, 800, 25

Wilson, C. D., Rangwala, N., Glenn, J., et al. 2014, ApJL, 789, L36 